\title{
DEMOGRAPHICS OF THE GALAXIES HOSTING SHORT-DURATION GAMMA-RAY BURSTS
}

\author{
W. Fong ${ }^{1}$, E. Berger ${ }^{1}$, R. Chornock ${ }^{1}$, R. Margutti ${ }^{1}$, A. J. Levan $^{2}$, N. R. TAnvir ${ }^{3}$, R. L. Tunnicliffe ${ }^{2}$, I. Czekala ${ }^{1}$, \\ D. B. Fox ${ }^{4}$, D. A. Perley ${ }^{5}$, S. B. Cenko ${ }^{6}$, B. A. Zauderer ${ }^{1}$, T. Laskar ${ }^{1}$, S. E. Persson ${ }^{7}$, A. J. Monson ${ }^{7}$, D. D. Kelson ${ }^{7}$,

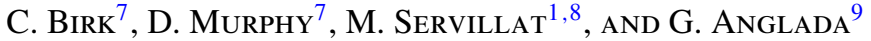 \\ ${ }^{1}$ Harvard-Smithsonian Center for Astrophysics, 60 Garden Street, Cambridge, MA 02138, USA \\ 2 Department of Physics, University of Warwick, Coventry CV4 7AL, UK \\ ${ }^{3}$ Department of Physics and Astronomy, University of Leicester, University Road, Leicester LE1 7RH, UK \\ ${ }^{4}$ Department of Astronomy and Astrophysics, 525 Davey Laboratory, Pennsylvania State University, University Park, PA 16802, USA \\ ${ }^{5}$ Cahill Center for Astronomy and Astrophysics, Room 232, California Institute of Technology, Pasadena, CA 91125, USA \\ ${ }^{6}$ Department of Astronomy, University of California, Berkeley, CA 94720-3411, USA \\ 7 Observatories of the Carnegie Institution for Science, 813 Santa Barbara Street, Pasadena, CA 91101, USA \\ ${ }^{8}$ Laboratoire AIM (CEA/DSM/IRFU/SAp, CNRS, Universite Paris Diderot), CEA Saclay, Bat. 709, F-91191 Gif-sur-Yvette, France \\ ${ }^{9}$ Institut für Astrophysik, Friedrich-Hund-Platz 1, Universität Göttingen, D-37077 Göttingen, Germany \\ Received 2013 January 7; accepted 2013 April 6; published 2013 May 3
}

\begin{abstract}
We present observations of the afterglows and host galaxies of three short-duration gamma-ray bursts (GRBs): 100625A, 101219A, and 110112A. We find that GRB 100625A occurred in a $z=0.452$ early-type galaxy with a stellar mass of $\approx 4.6 \times 10^{9} M_{\odot}$ and a stellar population age of $\approx 0.7 \mathrm{Gyr}$, and GRB 101219A originated in a star-forming galaxy at $z=0.718$ with a stellar mass of $\approx 1.4 \times 10^{9} M_{\odot}$, a star formation rate of $\approx 16 M_{\odot} \mathrm{yr}^{-1}$, and a stellar population age of $\approx 50$ Myr. We also report the discovery of the optical afterglow of GRB 110112A, which lacks a coincident host galaxy to $i \gtrsim 26 \mathrm{mag}$, and we cannot conclusively identify any field galaxy as a possible host. From afterglow modeling, the bursts have inferred circumburst densities of $\approx 10^{-4}-1 \mathrm{~cm}^{-3}$ and isotropic-equivalent gamma-ray and kinetic energies of $\approx 10^{50}-10^{51} \mathrm{erg}$. These three events highlight the diversity of galactic environments that host short GRBs. To quantify this diversity, we use the sample of 36 Swift short GRBs with robust associations to an environment $(\sim 1 / 2$ of 68 short bursts detected by Swift to 2012 May) and classify bursts originating from four types of environments: late-type $(\approx 50 \%)$, early-type $(\approx 15 \%)$, inconclusive $(\approx 20 \%)$, and "host-less" (lacking a coincident host galaxy to limits of $\gtrsim 26 \mathrm{mag} ; \approx 15 \%$ ). To find likely ranges for the true late- and early-type fractions, we assign each of the host-less bursts to either the late- or early-type category using probabilistic arguments and consider the scenario that all hosts in the inconclusive category are early-type galaxies to set an upper bound on the early-type fraction. We calculate most likely ranges for the late- and early-type fractions of $\approx 60 \%-80 \%$ and $\approx 20 \%-40 \%$, respectively. We find no clear trend between gamma-ray duration and host type. We also find no change to the fractions when excluding events recently claimed as possible contaminants from the long GRB/collapsar population. Our reported demographics are consistent with a short GRB rate driven by both stellar mass and star formation.
\end{abstract}

Key word: gamma-ray burst: general

Online-only material: color figures

\section{INTRODUCTION}

Observations of the galactic environments of cosmic explosions provide invaluable insight into their underlying progenitor populations. For example, Type Ia supernovae ( $\mathrm{SNe}$ ) originate in both star-forming and elliptical galaxies (Oemler \& Tinsley 1979; van den Bergh et al. 2005; Mannucci et al. 2005; Li et al. 2011) consistent with an evolved progenitor and an event rate that traces both stellar mass and star formation (Sullivan et al. 2006). In contrast, $\mathrm{SNe}$ of types $\mathrm{II}$ and $\mathrm{Ib} / \mathrm{c}$ are found to occur only in spiral and irregular galaxies, indicating that these events result from the core collapse of young, massive stars (van den Bergh et al. 2005; Hakobyan et al. 2008; Li et al. 2011) and a rate tracing recent star formation (Kelly \& Kirshner 2012; Anderson et al. 2012).

In the case of long-duration gamma-ray bursts (GRBs; $T_{90} \gtrsim$ $2 \mathrm{~s}$; Kouveliotou et al. 1993) the link to star-forming host galaxies helped to establish that their progenitors are massive stars (Djorgovski et al. 1998; Le Floc'h et al. 2003; Fruchter et al. 2006; Wainwright et al. 2007). Furthermore, a decade of concerted efforts to characterize the stellar populations of long
GRB hosts revealed young stellar population ages of $\leq 0.2 \mathrm{Gyr}$, a mean stellar mass of $\approx 2 \times 10^{9} M_{\odot}$, and inferred UV/optical star formation rates (SFRs) of $\approx 1-50 M_{\odot} \mathrm{yr}^{-1}$ (Christensen et al. 2004; Savaglio et al. 2009; Leibler \& Berger 2010; Laskar et al. 2011). In addition, the spatial locations of long GRBs with respect to their host galaxy centers (with a mean of $\sim 1$ half-light radius; Bloom et al. 2002) and their concentration in bright UV regions of their hosts (Fruchter et al. 2006) provided a direct association between long GRBs and star formation.

In contrast, the origin of short GRBs $\left(T_{90} \lesssim 2 \mathrm{~s}\right)$ is less clear, as the first few afterglow discoveries led to associations with both elliptical (Berger et al. 2005; Castro-Tirado et al. 2005; Gehrels et al. 2005; Hjorth et al. 2005a; Bloom et al. 2006) and star-forming (Fox et al. 2005; Hjorth et al. 2005b; Soderberg et al. 2006; Grupe et al. 2006; Burrows et al. 2006) host galaxies, demonstrating that at least some short GRBs originate from older stellar populations. Studies primarily focused on the sample of bursts with subarcsecond localization have shown the population of hosts to be dominated by late-type galaxies, albeit with lower specific SFRs, higher luminosities, and higher metallicities than the star-forming hosts of long GRBs (Berger 
2009). Modeling of the spectral energy distributions (SEDs) of short GRB host galaxies has led to a broad range of inferred ages, $\tau \approx 0.03-4.4 \mathrm{Gyr}$, and an average stellar mass of $\approx 2 \times$ $10^{10} M_{\odot}$ (Leibler \& Berger 2010). A detailed analysis of their sub-galactic environments through Hubble Space Telescope observations has demonstrated that on average, short GRBs have offsets from their hosts of $\approx 5 \mathrm{kpc}$ (Fong et al. 2010), while a growing subset that lack coincident hosts may have offsets of $\gtrsim 30 \mathrm{kpc}$ (Berger 2010a). Finally, an examination of short GRB locations with respect to their host light distributions revealed that they underrepresent their host UV/optical light (Fong et al. 2010). These results are consistent with theoretical expectations for NS-NS/NS-BH mergers (Eichler et al. 1989; Narayan et al. 1992), with potential minor contribution from other proposed progenitors, such as the accretion-induced collapse of a white dwarf or neutron star (NS) (Qin et al. 1998; Levan et al. 2006b; Metzger et al. 2008) or magnetar flares (Levan et al. 2006b; Chapman et al. 2008).

However, the majority of short GRB host galaxy studies published thus far primarily concentrate on bursts with subarcsecond localization from optical afterglows. While these events have the most unambiguous associations with host galaxies, the fraction is only $\sim 1 / 3$ (23/68 to 2012 May) of all short GRBs detected by the $S$ wift satellite (Gehrels et al. 2004). The faintness of their optical afterglows $(\approx 23 \mathrm{mag}$ at $\sim 10 \mathrm{hr}$ after the burst; Berger 2010a) is likely attributed to a combination of a low energy scale (Panaitescu et al. 2001) and circumburst densities. Therefore, if there exist correlations between these basic properties and host galaxy type, the selection by optical afterglows may affect the relative rates of short GRBs detected in early- and late-type host galaxies. An alternative route to subarcsecond localization is through the X-ray detection of an afterglow, which does not necessarily depend on circumburst density (Granot \& Sari 2002) with Chandra; however, only two such cases have been reported thus far (Fong et al. 2012; Margutti et al. 2012; Sakamoto et al. 2013).

Demographics that accurately represent the bulk of the short GRB population are imperative in understanding the link to the progenitors. In particular, the late-to-early-type host galaxy ratio will inform whether stellar mass or SFR drives the short GRB rate (Leibler \& Berger 2010) and will help to constrain the delay time distribution (Zheng \& Ramirez-Ruiz 2007). Furthermore, a recent study based on $\gamma$-ray properties (spectral hardness and duration) claims that there is a non-negligible fraction of contaminants from collapsars in the Swift short GRB population (Bromberg et al. 2013). Thus, an examination of how this fraction affects the environment demographics will aid in assessing the true contamination.

Fortunately, the detection of X-ray afterglows with Swift/XRT (Gehrels et al. 2004; Burrows et al. 2005) enables positions with $\sim$ few arcsecond precision in $\approx 60 \%(40 / 68)$ of all Swift short GRBs. In the majority of such cases, these X-Ray Telescope (XRT) positions coupled with dedicated optical/NIR searches for host galaxies have provided meaningful associations to a galactic environment. ${ }^{10}$ While such bursts with XRT positions have been studied as single events (e.g., Gehrels et al. 2005; Bloom et al. 2006, 2007; Perley et al. 2012), the entire sample has not been studied in detail alongside bursts with subarcsecond localization.

\footnotetext{
10 The large majority of the remaining $\approx 40 \%$ of Swift short GRBs lack afterglow follow-up due to observing constraints unrelated to the burst properties; see Section 5.
}

To this end, we present here X-ray and optical/NIR observations of the afterglows and environments of three short GRBs $^{11}$ localized by Swift/XRT, which highlight the diversity of their galactic environments: GRBs 100625A, 101219A, and $110112 \mathrm{~A}$. We also present the discovery of the optical afterglow of GRB 110112A. While GRBs 100625A and 101219A have robust associations with host galaxies, GRB 110112A lacks a coincident host to deep optical limits. We describe the X-ray, optical, and NIR observations for these three events (Section 2), present their energy scales and circumburst densities inferred from afterglow modeling (Section 3), and provide host galaxy stellar population ages, masses, and SFRs extracted from spectroscopy and broadband SEDs (Section 4). We discuss the stellar population characteristics of these three host galaxies compared to previous short GRB hosts (Section 5). Putting these bursts into context, we undertake the first comprehensive study of host galaxy demographics of both subarcsecond localized and XRT-localized bursts, by investigating the late- and early-type host galaxy fractions for the bulk of the short GRB population, and compare host galaxy type to $\gamma$-ray properties (Section 6).

Unless otherwise noted, all magnitudes are in the AB system and are corrected for Galactic extinction in the direction of the burst (Schlegel et al. 1998; Schlafly \& Finkbeiner 2011), and uncertainties correspond to $1 \sigma$ confidence. We employ a standard $\Lambda$ CDM cosmology with $\Omega_{M}=0.27, \Omega_{\Lambda}=0.73$, and $H_{0}=71 \mathrm{~km} \mathrm{~s}^{-1} \mathrm{Mpc}^{-1}$.

\section{OBSERVATIONS}

\subsection{GRB $100625 A$}

GRB 100625A was detected by three $\gamma$-ray satellites on 2010 June 25.773 UT: the Burst Alert Telescope (BAT) on board the Swift satellite (Gehrels et al. 2004; Holland et al. 2010a), Konus-Wind (Golenetskii et al. 2010a), and the GammaRay Burst Monitor (GBM) on board Fermi (Bhat 2010). BAT localized the burst to a ground-calculated position of R.A. = $01^{\mathrm{h}} 03^{\mathrm{m}} 11^{\mathrm{s}} .1$, decl. $=-39^{\circ} 05^{\prime} 29^{\prime \prime}(\mathrm{J} 2000)$ with an uncertainty of 1.0 radius (90\% containment; Holland et al. 2010b), and the burst consisted of two pulses with a total duration of $T_{90}=0.33 \pm 0.03 \mathrm{~s}(15-350 \mathrm{keV})$ and a fluence of $f_{\gamma}=(2.3 \pm$ $0.2) \times 10^{-7} \mathrm{erg} \mathrm{cm}^{-2}$ (15-150 keV; Holland et al. 2010b). Fermi/ GBM observations determined $E_{\text {peak }}=509_{-61}^{+77} \mathrm{keV}$ and $f_{\gamma}=$ $(1.32 \pm 0.05) \times 10^{-6} \mathrm{erg} \mathrm{cm}^{-2}(8-1000 \mathrm{keV}$; Bhat 2010), while Konus - Wind observations determined $E_{\text {peak }}=418_{-78}^{+128} \mathrm{keV}$ and $f_{\gamma}=(8.3 \pm 1.5) \times 10^{-7} \mathrm{erg} \mathrm{cm}^{-2}(20-2000 \mathrm{keV}$; Golenetskii et al. 2010a). Based on the short duration and high $E_{\text {peak }}$, GRB 100625A can be classified as a short, hard burst. The $\gamma$-ray properties are listed in Table 1 .

\subsubsection{X-Ray Observations}

The XRT on board Swift began observing the field at $\delta t=43 \mathrm{~s}$ ( $\delta t$ is the time after the BAT trigger) and detected a fading, uncataloged X-ray source at R.A. $=01^{\mathrm{h}} 03^{\mathrm{m}} 10^{\mathrm{s}} .91$ and decl. $=$ $-39^{\circ} 05^{\prime} 18^{\prime \prime} .4$ with a final positional accuracy of $1^{\prime \prime} .8$ radius (90\%; Goad et al. 2007; Evans et al. 2009; Holland et al. 2010b; Table 1).

\footnotetext{
11 We present observations of two additional short GRBs, 100628A and 100702A, both with published Swift/XRT localizations (see Appendices A and B). We show that the XRT afterglow of GRB 100628A is of low significance, while the XRT position of GRB 100702A is contaminated, preventing an unambiguous association with a host galaxy.
} 
Table 1

Short GRB Properties

\begin{tabular}{|c|c|c|c|c|c|c|c|}
\hline GRB & $\begin{array}{c}\text { R.A. } \\
(\mathrm{J} 2000)\end{array}$ & $\begin{array}{c}\text { Decl. } \\
(\mathrm{J} 2000)\end{array}$ & $\begin{array}{l}\text { Uncert. } \\
\text { (") }\end{array}$ & $z$ & $\begin{array}{c}T_{90}(15-350 \mathrm{keV}) \\
(\mathrm{s})\end{array}$ & $\begin{array}{c}f_{\gamma}(15-150 \mathrm{keV}) \\
\quad\left(\operatorname{erg~cm}^{-2}\right)\end{array}$ & References \\
\hline GRB 100625A & $01^{\mathrm{h}} 03^{\mathrm{m}} 10^{\mathrm{s}} .91$ & $-39^{\circ} 05^{\prime} 18^{\prime \prime} .4$ & 1.8 & 0.452 & $0.33 \pm 0.03$ & $(2.3 \pm 0.2) \times 10^{-7}$ & 1 \\
\hline GRB 101219A & $04^{\mathrm{h}} 58^{\mathrm{m}} 20.49$ & $-02^{\circ} 32^{\prime} 23^{\prime \prime} .0$ & 1.7 & 0.718 & $0.6 \pm 0.2$ & $(4.6 \pm 0.3) \times 10^{-7}$ & 2 \\
\hline GRB 110112A & $21^{\mathrm{h}} 59^{\mathrm{m}} 43^{\mathrm{s}} .85$ & $+26^{\circ} 27^{\prime} 23^{\prime \prime} .9$ & 0.14 & $\ldots$ & $0.5 \pm 0.1$ & $(3.0 \pm 0.9) \times 10^{-8}$ & 3 , This work \\
\hline
\end{tabular}

References. (1) Holland et al. 2010b; (2) Krimm et al. 2010a; (3) Barthelmy et al. 2011.

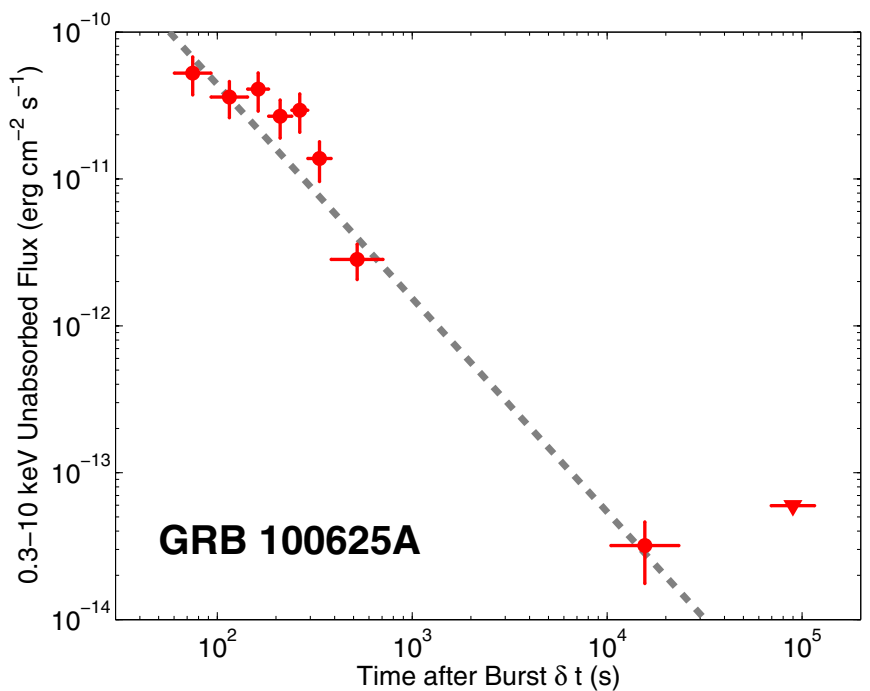

Figure 1. Swift/XRT light curve of GRB 100625A. The triangle is a $3 \sigma$ upper limit. The entire light curve is best fit with a power law characterized by $\alpha_{X}=-1.45 \pm 0.08$ (gray dashed line).

(A color version of this figure is available in the online journal.)

We analyze the XRT data using HEASOFT (ver. 6.11) and relevant calibration files. We apply standard filtering and screening criteria and generate a count rate light curve following the prescriptions from Margutti et al. (2010) and Margutti et al. (2013). Our re-binning scheme ensures a minimum signal-tonoise ratio of $\mathrm{S} / \mathrm{N}=4$ for each temporal bin. To extract a spectrum, we use Cash statistics and fit the XRT data with an absorbed power-law model (tbabs $\times$ ztbabs $\times$ pow within the XSPEC routine) characterized by photon index, $\Gamma$, and intrinsic neutral hydrogen absorption column density, $N_{\mathrm{H} \text {,int }}$, in excess of the Galactic column density in the direction of the burst, $N_{\mathrm{H}, \mathrm{MW}}=2.1 \times 10^{20} \mathrm{~cm}^{-2}$ (typical uncertainty of $\sim 10 \%$; Kalberla et al. 2005; Wakker et al. 2011). We utilize the entire PC data set $\left(\delta t=60-10^{5} \mathrm{~s}\right)$, where there is no evidence for spectral evolution. Our best-fit spectrum $\left(C\right.$-stat $_{v}=0.92$ for $95 \mathrm{dof}$ ) is characterized by $\Gamma=2.5 \pm 0.2$ and $N_{\mathrm{H} \text {,int }} \lesssim$ $1.7 \times 10^{21} \mathrm{~cm}^{-2}(3 \sigma)$ at $z=0.452$ (see Section 4.1 for the redshift determination). Our best-fit parameters are consistent with the automatic spectrum fit produced by Page \& Holland (2010). Applying these parameters to the data, we calculate the count rate-to-flux conversion factors, and hence the unabsorbed fluxes (Figure 1).

To quantify the decay rate, we utilize $\chi^{2}$-minimization to fit a power law to the data in the form $F_{X}(t) \propto t^{\alpha_{X}}$, with $\alpha_{X}$ as the free parameter. The entire XRT light curve $\left(\delta t \approx 80-10^{5} \mathrm{~s}\right.$, PC mode) is best fit with a single power law with index $\alpha_{X}=-1.45 \pm 0.08$ $\left(\chi_{v}^{2}=2.1\right.$ for 7 dof; Figure 1$)$.

\subsubsection{Optical/NIR Observations and Afterglow Limits}

The UV-Optical Telescope (UVOT) on board Swift commenced observations at $\delta t=56 \mathrm{~s}$, but no corresponding source was found within the XRT position. The $3 \sigma$ limit over $\delta t \approx 87-1.2 \times 10^{4} \mathrm{~s}$ in the white filter, which transmits over $\lambda=1600-7000 \AA$ (Poole et al. 2008), is $\gtrsim 22.6 \mathrm{mag}$ (not corrected for Galactic extinction; Holland et al. 2010b). Rapid ground-based follow-up in the optical and NIR provided early limits on the afterglow of $I \gtrsim 22.8 \mathrm{mag}$ at $\delta t \approx 17$ minutes (Suzuki et al. 2010) and $J \gtrsim 19.4 \mathrm{mag}$ at $\delta t \approx 8.6 \mathrm{hr}$ (Naito et al. 2010). GROND observations at $\delta t \approx 12.2 \mathrm{hr}$ place limits of $g \gtrsim 23.6$ mag and riz $\gtrsim 23$ mag (Nicuesa Guelbenzu et al. 2012).

We obtained optical observations of GRB 100625A with the Gemini Multi-Object Spectrograph (GMOS) mounted on the Gemini-South $8 \mathrm{~m}$ telescope, starting at $\delta t=12.4 \mathrm{hr}$ in the riz filters in poor seeing conditions (Table 2 ). We analyze the data using the IRAF gemini package and detect a single source within the enhanced XRT error circle in all three filters. To assess any potential fading of the source, we obtained a second set of observations at $\delta t \approx 2.6$ days, where the source is clearly extended. Digital image subtraction using the ISIS software package (Alard 2000) shows no residuals in all three filters (Figure 2). We therefore place $3 \sigma$ limits of $r \gtrsim 22.6 \mathrm{mag}$, $i \gtrsim 22.7 \mathrm{mag}$, and $z \gtrsim 22.8 \mathrm{mag}$ on the optical afterglow at $\delta t \approx 12.7 \mathrm{hr}$ (Table 2 ). The GMOS zero points are determined by sources in common with late-time Inamori Magellan Areal Camera and Spectrograph (IMACS) observations (see below), which are calibrated to a standard star field at a similar airmass. Our limits match the GROND limits reported at $\delta t \approx 12.2 \mathrm{hr}$ (Nicuesa Guelbenzu et al. 2012).

In addition, we obtained two epochs of $J$-band observations with the Persson's Auxiliary Nasmyth Infrared Camera (PANIC) mounted on the $6.5 \mathrm{~m}$ Magellan/Baade telescope at $\delta t \approx 1.6$ and 6.6 days. We analyze the data using standard procedures in IRAF. Digital image subtraction shows no evidence for fading, with a $3 \sigma$ limit of $J \gtrsim 23.9$ mag (photometrically tied to the Two Micron All Sky Survey (2MASS) catalog and converted to the $\mathrm{AB}$ system) at $\delta t \approx 1.6$ days (Table 2 ).

We obtained late-time griz observations of the field of GRB 100625A with the IMACS mounted on Magellan/Baade starting on 2010 November 14.11 UT. We also obtained $K_{s^{-}}$ band observations with the FourStar Infrared Camera mounted on Magellan/Baade on 2011 December 07.16 UT (Table 2). The griz zero points are calculated using a standard star field at a similar airmass, while the $K_{s}$-band zero point is determined from point sources in common with 2 MASS. Our afterglow limit and host galaxy photometry are summarized in Table 2.

We obtained a spectrum of the putative host galaxy with the Low Dispersion Survey Spectrograph 3 (LDSS3) mounted on the $6.5 \mathrm{~m}$ Magellan/Clay telescope on 2011 October 


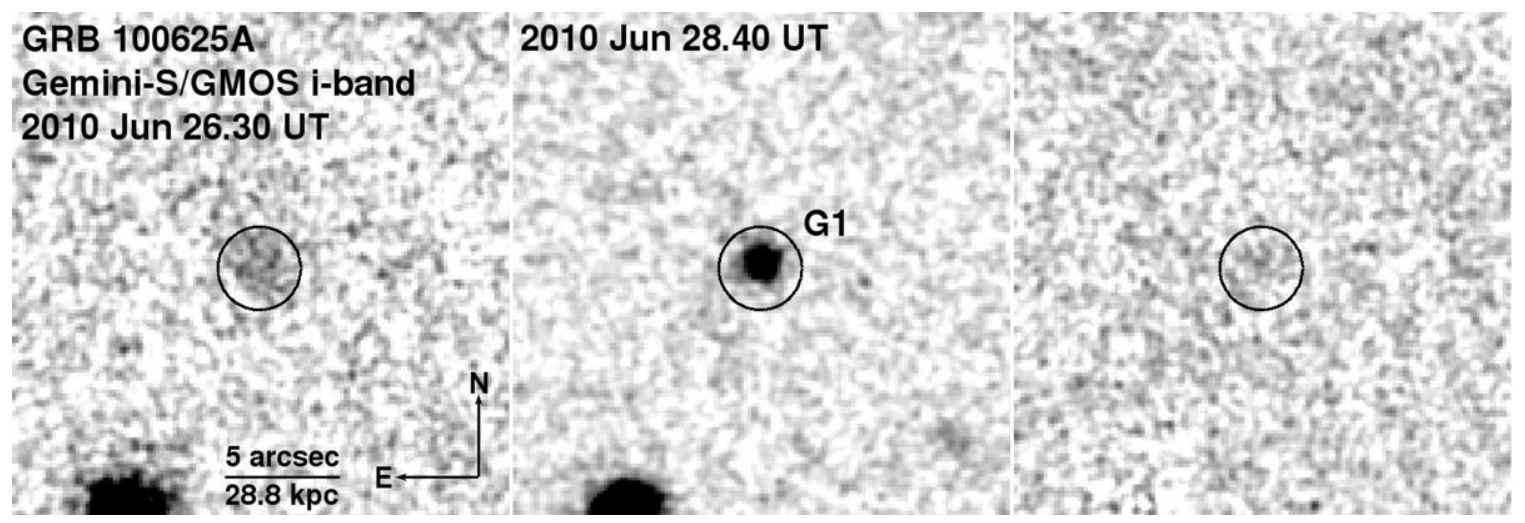

Figure 2. Gemini-South/GMOS $i$-band observations of GRB 100625A. The XRT error circle has a radius of 1".8 (90\% containment; black). Images are smoothed with a 2-pixel Gaussian. Left: $\delta t=0.53$ days in poor seeing conditions $\left(\theta_{\mathrm{FWHM}}=1^{\prime \prime} \cdot 9\right)$ with a faint host detection. Center: $\delta t=2.63$ days with $0^{\prime \prime} \cdot 9$ seeing. Right: digital image subtraction of the two epochs reveals no afterglow to a $3 \sigma$ limit of $i \gtrsim 22.7 \mathrm{mag}$. The host galaxy is marked as G1.

Table 2

Log of Optical/NIR Afterglow and Host Galaxy Photometry

\begin{tabular}{|c|c|c|c|c|c|c|c|c|c|c|c|}
\hline GRB & $\begin{array}{l}\text { Date } \\
\text { (UT) }\end{array}$ & $\begin{array}{l}\delta t \\
\text { (d) }\end{array}$ & Telescope & Instrument & Filter & $\begin{array}{c}\text { Exposures } \\
\text { (s) }\end{array}$ & $\begin{array}{c}\theta_{\text {FWHM }} \\
\left({ }^{\prime \prime}\right)\end{array}$ & $\begin{array}{l}\text { Afterglow }^{\mathrm{a}} \\
\text { (AB mag) }\end{array}$ & $\begin{array}{c}F_{v}{ }^{\mathrm{a}} \\
(\mu \mathrm{Jy})\end{array}$ & $\begin{array}{c}\text { Host }^{\mathrm{a}} \\
(\mathrm{AB} \mathrm{mag})\end{array}$ & $\begin{array}{r}A_{\lambda, \mathrm{MW}} \\
(\mathrm{mag})\end{array}$ \\
\hline \multirow[t]{13}{*}{ GRB 100625A } & 2010 Jun 26.288 & 0.52 & Gemini-S & GMOS & $r$ & $5 \times 120$ & 2.31 & $>22.6$ & $<3.3$ & $22.76 \pm 0.23$ & 0.027 \\
\hline & 2010 Jun 26.301 & 0.53 & Gemini-S & GMOS & $i$ & $3 \times 120$ & 1.91 & $>22.7$ & $<2.9$ & $22.10 \pm 0.15$ & 0.020 \\
\hline & 2010 Jun 26.314 & 0.54 & Gemini-S & GMOS & $z$ & $5 \times 120$ & 1.95 & $>22.8$ & $<2.8$ & $22.23 \pm 0.15$ & 0.015 \\
\hline & 2010 Jun 27.392 & 1.62 & Magellan & PANIC & $J$ & $35 \times 60$ & 0.76 & $>23.9$ & $<1.0$ & $21.48 \pm 0.05$ & 0.008 \\
\hline & 2010 Jun 28.394 & 2.62 & Gemini-S & GMOS & $r$ & $5 \times 120$ & 1.10 & & & $22.63 \pm 0.09$ & 0.027 \\
\hline & 2010 Jun 28.404 & 2.63 & Gemini-S & GMOS & $i$ & $5 \times 120$ & 0.87 & & & $22.14 \pm 0.04$ & 0.020 \\
\hline & 2010 Jun 28.414 & 2.64 & Gemini-S & GMOS & $z$ & $5 \times 120$ & 0.95 & & & $22.07 \pm 0.10$ & 0.015 \\
\hline & $2010 \mathrm{Jul} 02.398$ & 6.63 & Magellan & PANIC & $J$ & $18 \times 180$ & 0.53 & & & $21.40 \pm 0.06$ & 0.008 \\
\hline & 2010 Nov 14.114 & 141.3 & Magellan & IMACS & $g$ & $2 \times 420$ & 0.65 & & & $23.87 \pm 0.19$ & 0.039 \\
\hline & 2010 Nov 14.123 & 141.4 & Magellan & IMACS & $i$ & $1 \times 240$ & 0.47 & & & $22.04 \pm 0.07$ & 0.020 \\
\hline & 2010 Nov 14.196 & 141.4 & Magellan & IMACS & $r$ & $1 \times 360$ & 0.65 & & & $22.59 \pm 0.13$ & 0.027 \\
\hline & 2010 Nov 14.200 & 141.4 & Magellan & IMACS & $z$ & $1 \times 180$ & 0.52 & & & $21.88 \pm 0.22$ & 0.015 \\
\hline & 2011 Dec 07.16 & 529.4 & Magellan & FourStar & $K_{s}$ & $90 \times 10$ & 0.55 & & & $20.76 \pm 0.10$ & 0.008 \\
\hline \multirow[t]{3}{*}{ GRB 100702A } & $2010 \mathrm{Jul} 02.10$ & 0.05 & Magellan & PANIC & $J$ & $9 \times 180$ & 0.53 & $>23.3^{\mathrm{b}}$ & $<1.70^{\mathrm{b}}$ & $20.54 \pm 0.05 / 21.30 \pm 0.07^{\mathrm{c}}$ & 0.284 \\
\hline & $2010 \mathrm{Jul} 02.30$ & 0.25 & Magellan & PANIC & $J$ & $9 \times 180$ & 0.75 & & & $\ldots \mathrm{d} / 21.49 \pm 0.11$ & 0.284 \\
\hline & 2011 Mar 06.37 & 247.3 & Magellan & IMACS & $i$ & $2 \times 240$ & 0.83 & & & $>22.7$ & 0.679 \\
\hline \multirow[t]{11}{*}{ GRB 101219A } & 2010 Dec 19.15 & 0.04 & Gemini-S & GMOS & $i$ & $9 \times 180$ & 0.66 & $>24.9$ & $<0.40$ & $23.20 \pm 0.11$ & 0.097 \\
\hline & 2010 Dec 19.16 & 0.05 & Magellan & FourStar & $J$ & $25 \times 60$ & 0.46 & $>23.6$ & $<1.36$ & $22.43 \pm 0.13$ & 0.041 \\
\hline & 2010 Dec 19.17 & 0.07 & Gemini-S & GMOS & $r$ & $9 \times 180$ & 0.80 & $>24.9$ & $<0.40$ & $23.83 \pm 0.26$ & 0.131 \\
\hline & 2010 Dec 19.20 & 0.09 & Gemini-S & GMOS & $i$ & $9 \times 180$ & 0.69 & $>24.9$ & $<0.40$ & $23.40 \pm 0.09$ & 0.097 \\
\hline & 2010 Dec 19.27 & 0.16 & Gemini-S & GMOS & $r$ & $12 \times 180$ & 0.67 & $>25.1$ & $<0.34$ & $23.73 \pm 0.10$ & 0.131 \\
\hline & 2010 Dec 19.30 & 0.20 & Gemini-S & GMOS & $i$ & $12 \times 180$ & 0.67 & & & $23.19 \pm 0.08$ & 0.097 \\
\hline & 2010 Dec 28.16 & 9.05 & Gemini-S & GMOS & $r$ & $12 \times 240$ & 0.65 & & & $23.95 \pm 0.05$ & 0.131 \\
\hline & 2011 Jan 12.15 & 24.05 & Magellan & LDSS3 & $z$ & $6 \times 180$ & 0.68 & & & $23.22 \pm 0.16$ & 0.072 \\
\hline & 2011 Jan 12.17 & 24.06 & Magellan & LDSS3 & $g$ & $5 \times 180$ & 1.05 & & & $24.57 \pm 0.08$ & 0.189 \\
\hline & 2011 Dec 07.24 & 353.1 & Magellan & FourStar & $J$ & $15 \times 60$ & 0.56 & & & $22.11 \pm 0.19$ & 0.041 \\
\hline & 2011 Dec 07.25 & 353.1 & Magellan & FourStar & $K_{s}$ & $90 \times 10$ & 0.44 & & & $21.55 \pm 0.21$ & 0.017 \\
\hline \multirow[t]{4}{*}{ GRB 110112A } & 2011 Jan 12.18 & 0.64 & WHT & ACAM & $i$ & $2 \times 300$ & 1.10 & $22.77 \pm 0.29$ & $2.84 \pm 0.75$ & $\ldots$ & 0.104 \\
\hline & 2011 Jun 27.83 & 166.2 & Magellan & LDSS3 & $i$ & $5 \times 240$ & 0.94 & & & $>24.7$ & 0.104 \\
\hline & 2011 Jun 27.83 & 166.3 & Magellan & LDSS3 & $r$ & $3 \times 360$ & 1.11 & & & $>25.5$ & 0.140 \\
\hline & $2011 \mathrm{Jul} 28.46$ & 197.3 & Gemini-N & GMOS & $i$ & $15 \times 180$ & 0.61 & & & $>26.2$ & 0.104 \\
\hline
\end{tabular}

Notes. Limits correspond to a $3 \sigma$ confidence level.

a These values are corrected for Galactic extinction (Schlafly \& Finkbeiner 2011).

${ }^{\mathrm{b}}$ Only applies to approximately half of the error circle.

${ }^{c}$ Magnitudes for S1 and S4, respectively.

${ }^{\mathrm{d}} \mathrm{S} 1$ is blended with a neighboring bright star (Figure 16) so we cannot perform photometry.

21.27 UT. A dithered pair of $2700 \mathrm{~s}$ exposures was obtained with the VPH-ALL grating, which has a wavelength coverage of $4000-10000 \AA$ and a spectral resolution of $\approx 8 \AA$. We used standard tasks in IRAF for data reduction, $\mathrm{HeNeAr}$ arc lamps for wavelength calibration, and observations of the smooth-spectrum standard star EG131 for flux calibration. We discuss the spectral features and redshift determination in Section 4.1. 


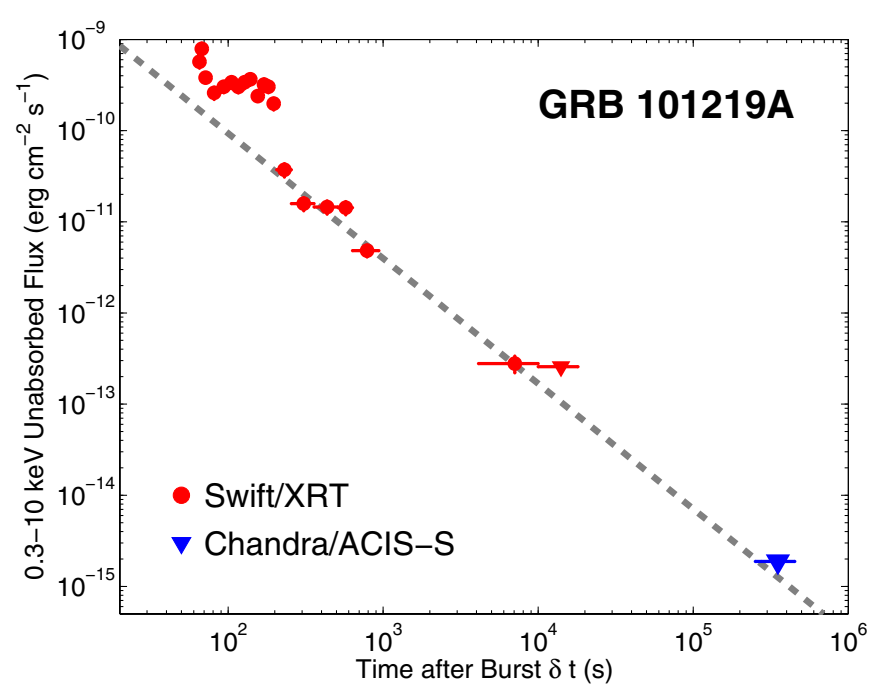

Figure 3. X-ray afterglow light curve of GRB 101219A, including Swift/XRT observations (red points) and a Chandra/ACIS-S observation (blue point). Triangles denote $3 \sigma$ upper limits. The data over $\delta t \approx 200-10^{4} \mathrm{~s}$ are best fit with a power law characterized by $\alpha_{X}=-1.37 \pm 0.13$ (gray dashed line).

(A color version of this figure is available in the online journal.)

\subsection{GRB $101219 A$}

GRB 101219A was detected by Swift/BAT (Gelbord et al. 2010) and Konus-Wind (Golenetskii et al. 2010b) on 2010 December 19.105 UT. BAT localized the burst at a groundcalculated position of R.A. $=04^{\mathrm{h}} 58^{\mathrm{m}} 20^{\mathrm{s}} .7$ and decl. $=$ $-02^{\circ} 31^{\prime} 37^{\prime \prime} .1$ with a $1^{\prime} .0$ radius uncertainty $(90 \%$ containment; Krimm et al. 2010a). The $\gamma$-ray light curve exhibits a doublepeaked structure with $T_{90}=0.6 \pm 0.2 \mathrm{~s}(15-350 \mathrm{keV})$ and $f_{\gamma}=(4.6 \pm 0.3) \times 10^{-7} \mathrm{erg} \mathrm{cm} \mathrm{cm}^{-2}(15-150 \mathrm{keV} ;$ Krimm et al. 2010a). Konus - Wind observations determined $E_{\text {peak }}=$ $490_{-79}^{+103} \mathrm{keV}$ and $f_{\gamma}=(3.6 \pm 0.5) \times 10^{-6} \mathrm{erg} \mathrm{cm}^{-2}\left(20-10^{4} \mathrm{keV}\right.$; Golenetskii et al. 2010b). Based on the short duration and high $E_{\text {peak }}$, GRB 101219A can be classified as a short, hard burst. The $\gamma$-ray properties are listed in Table 1 .

\subsubsection{X-Ray Observations}

Swift/XRT began observing the field at $\delta t=40 \mathrm{~s}$ and detected a fading, uncataloged X-ray source at R.A. $=04^{\mathrm{h}} 58^{\mathrm{m}} 20.49$ and decl. $=-02^{\circ} 32^{\prime} 23^{\prime \prime} .0$ with final accuracy of $1^{\prime \prime} .7$ (Goad et al. 2007; Evans et al. 2009; Table 1). We re-bin the XRT data and extract the best-fit spectrum for GRB 101219A as described in Section 2.1.1. We utilize the PC data set, $\delta t=70-10^{4} \mathrm{~s}$, where there is no evidence for spectral evolution. We find an average best-fitting spectrum characterized by $\Gamma=1.8 \pm 0.1$ and $N_{\mathrm{H}, \text { int }}=6.6_{-1.8}^{+2.3} \times 10^{21} \mathrm{~cm}^{-2}$ at $z=0.718\left(C-\right.$ stat $_{v}=0.97$ for 211 dof; see Section 4.2 for redshift determination) in excess of the Galactic absorption, $N_{\mathrm{H}, \mathrm{MW}}=4.9 \times 10^{20} \mathrm{~cm}^{-2}$ (Kalberla et al. 2005). Our best-fit parameters are consistent with the automatic spectrum fit produced by Gelbord \& Grupe (2010). Applying these parameters to the XRT data, we calculate the count rate-to-flux conversion factors, and hence the unabsorbed fluxes (Figure 3).

In addition, we obtained a $20 \mathrm{ks}$ observation with the Advanced CCD Imaging Spectrometer (ACIS-S) on board the Chandra X-Ray Observatory starting at $\delta t=4.1$ days. We analyze the Chandra data with the CIAO data reduction package. In an energy range of $0.5-8 \mathrm{keV}$, we extract four counts in a 2.5 aperture centered on the XRT position, consistent with the average $3 \sigma$ background level calculated from sourcefree regions on the same chip. We take this count rate of $\lesssim 2 \times 10^{-4}$ counts $\mathrm{s}^{-1}$ to be the $3 \sigma$ upper limit on the X-ray afterglow flux at $\delta t \approx 4.1$ days. Applying the spectrum extracted from the XRT data, this count rate corresponds to $F_{X} \lesssim 1.9 \times 10^{-15} \mathrm{erg} \mathrm{cm}^{-2} \mathrm{~s}^{-1}$.

The $\mathrm{X}$-ray light curve is characterized by a steep decay and a short plateau for $\delta t<200 \mathrm{~s}$, followed by a steady decline to the end of XRT observations at $\delta t \approx 10^{4} \mathrm{~s}$. To quantify this decay rate, we utilize the single-parameter $\chi^{2}$-minimization method described in Section 2.1.1. Excluding the XRT data at $\delta t \lesssim 200 \mathrm{~s}$ and the late-time upper limits, the best-fit power-law index is $\alpha_{X}=-1.37 \pm 0.13\left(\chi_{v}^{2}=1.1\right.$ for 5 dof $)$. The full X-ray afterglow light curve, along with the best-fit model, is shown in Figure 3.

\subsubsection{Optical/NIR Observations and Afterglow Limits}

UVOT commenced observations at $\delta t=67 \mathrm{~s}$. Over $\delta t=$ 67-5500 s, no corresponding source was found within the XRT position to a $3 \sigma$ limit of $\gtrsim 21.4$ in the white filter (Kuin \& Gelbord 2010).

We observed the field of GRB 101219A in both $r$ and $i$ bands with GMOS on Gemini-South, and in $J$ band with FourStar, starting at $\delta t \approx 0.96 \mathrm{hr}$ (Table 2 ). We detect a single extended source within the XRT error circle in all filters. To assess any fading, we obtained additional observations in the $r i$ bands at $\delta t \approx 0.2$ days (Table 2). Digital image subtraction between these epochs does not reveal any residuals (Figure 4), allowing us to place limits on the optical afterglow of $i \gtrsim 24.9$ mag and $r \gtrsim 24.9 \mathrm{mag}$ at the time of the first epoch for each filter: $\delta t \approx 0.96$ and $2.2 \mathrm{hr}$, respectively (Table 2 ). To assess the fading on timescales $\gtrsim 1$ day, we obtained a third set of observations in the $r$ band at $\delta t \approx 9$ days. Image subtraction with each of the first and second $r$-band observations also shows no evidence for fading (Table 2). A second set of $J$-band observations at $\delta t \approx 350$ days and a clean image subtraction with the first epoch allows us to place a limit on the NIR afterglow of $J \gtrsim 23.6 \mathrm{mag}$ at $\delta t=1.7 \mathrm{hr}$. Finally, to complement our early optical/NIR observations, we obtained imaging of the putative host galaxy in the $g z$ bands with LDSS3 starting on 2011 January 12.15 UT, and in the $K_{s}$ band with FourStar on 2011 December 07.24 UT. Our limits for the afterglow and photometry of the putative host galaxy are summarized in Table 2.

We obtained spectroscopic observations of the host on 2011 January 2.25 UT using GMOS on Gemini-North at a mean airmass of 1.2. We obtained a set of $4 \times 1800 \mathrm{~s}$ exposures with the R400 grating and an order-blocking filter, OG515 in the nod-and-shuffle mode, covering 5860-10200 $\AA$ at a spectral resolution of $\approx 7 \AA$. We used standard tasks in IRAF for data reduction, $\mathrm{CuAr}$ arc lamps for wavelength calibration, and archival observations of the smooth-spectrum standard star BD+28 4211 for flux calibration. We discuss the characteristics of the spectrum and redshift determination in Section 4.2.

\subsection{GRB $110112 A$}

Swift/BAT detected GRB 110112A on 2011 January 12.175 UT (Stamatikos et al. 2011), with a single spike with $T_{90}=0.5 \pm 0.1 \mathrm{~s}(15-350 \mathrm{keV})$ and $f_{\gamma}=(3.0 \pm 0.9) \times$ $10^{-8} \mathrm{erg} \mathrm{cm}^{-2}$ (15-150 keV; Barthelmy et al. 2011). The BAT ground-calculated position is R.A. $=21^{\mathrm{h}} 59^{\mathrm{m}} 33.6$ and decl. $=$ $+26^{\circ} 28^{\prime} 10^{\prime} .6$ with $2^{\prime} .6$ radius uncertainty $(90 \%$ containment; Barthelmy et al. 2011). The $\gamma$-ray properties are listed in Table 1. 


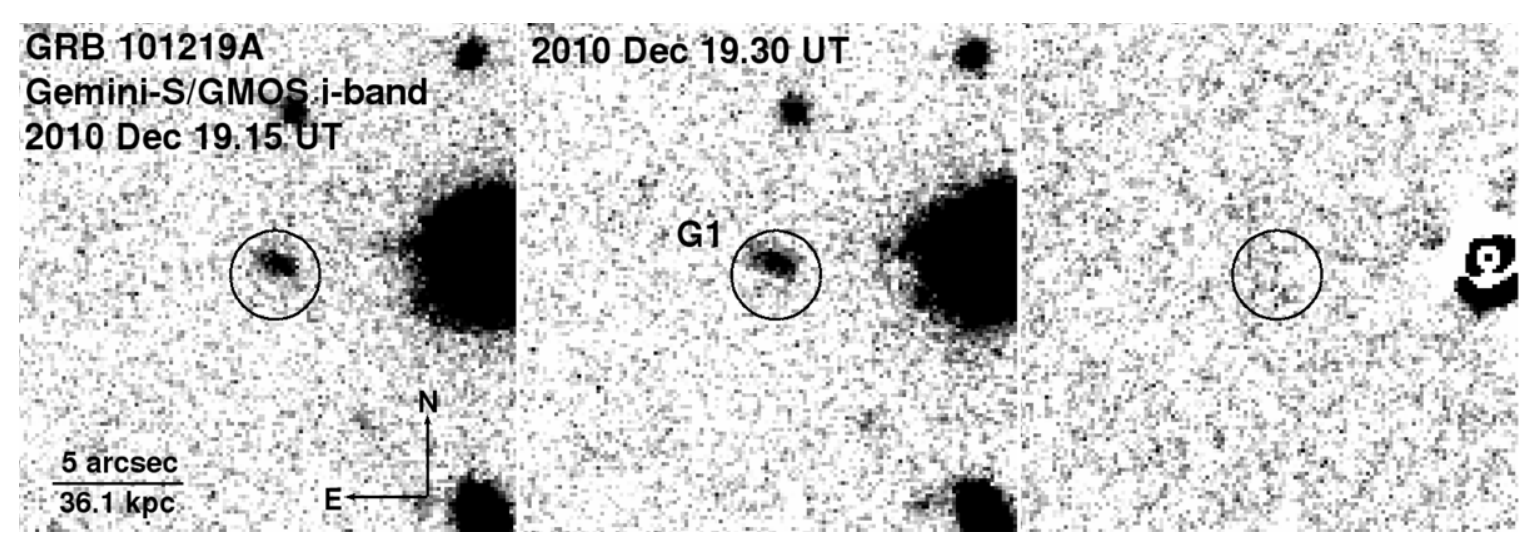

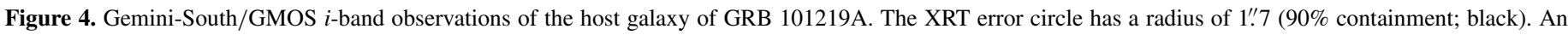

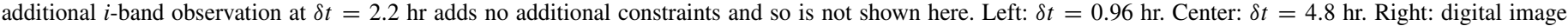
subtraction of the two epochs reveals no afterglow to a $3 \sigma$ limit of $i \gtrsim 24.9$ mag

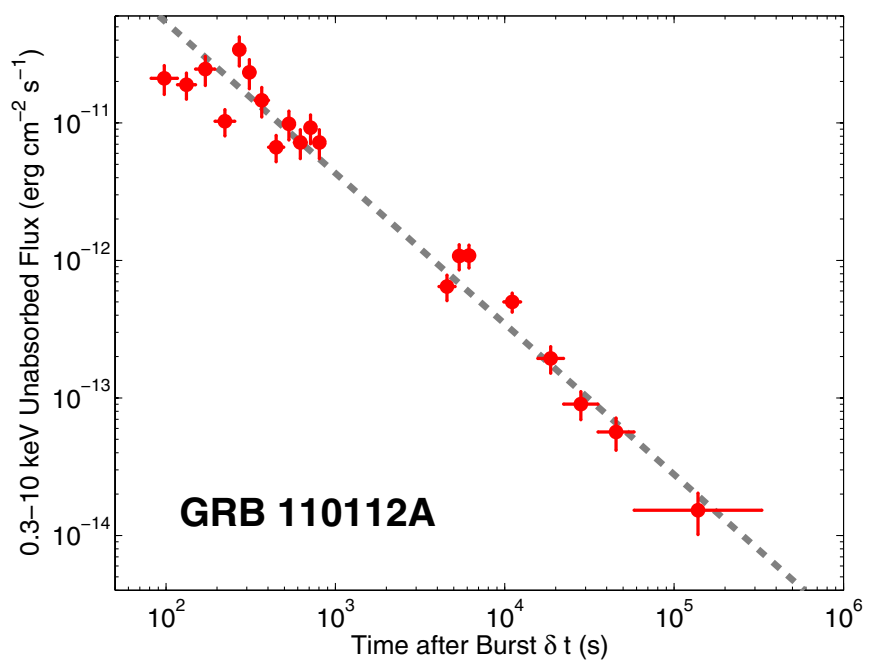

Figure 5. Swift/XRT light curve of GRB 110112A. The data (red points) for $\delta t \gtrsim 200 \mathrm{~s}$ are best fit with a single power law characterized by $\alpha_{X}=-\widetilde{1.10} \pm 0.05$ (gray dashed line).

(A color version of this figure is available in the online journal.)

\subsubsection{X-Ray Observations}

XRT commenced observations of the field of GRB 110112A at $\delta t=76 \mathrm{~s}$ and located a fading X-ray counterpart with a UVOT-enhanced positional accuracy of 1".6 radius (Evans et al. 2009, 2011; Goad et al. 2007; Table 1). We extract the XRT light curve and spectrum in the manner described in Section 2.1.1, requiring a minimum $\mathrm{S} / \mathrm{N}=3$ for each bin, and use the Galactic absorption in the direction of the burst of $N_{\mathrm{H}, \mathrm{MW}}=5.5 \times 10^{20} \mathrm{~cm}^{-2}$ (Kalberla et al. 2005). The light curve is characterized by a short plateau for $\delta t \lesssim 200 \mathrm{~s}$, followed by a steady decline (Figure 5). Performing $\chi^{2}$-minimization, we find the XRT light curve for $\delta t \gtrsim 200 \mathrm{~s}$ is best fit with a single power law characterized by index $\alpha_{X}=-1.10 \pm 0.05\left(\chi_{v}^{2}=1.0\right.$ for $17 \mathrm{dof}$ ). Our best-fitting spectral parameters over the entire data set, where there is no evidence for spectral evolution, are $\Gamma=2.2 \pm 0.2$ and an upper limit of $N_{\mathrm{H} \text {,int }} \lesssim 1.6 \times 10^{21} \mathrm{~cm}^{-2}$ ( $3 \sigma$ at $z=0 ; C$-stat $=0.82$ for 156 dof).

\subsubsection{Optical Afterglow Discovery}

UVOT commenced observations at $\delta t=80 \mathrm{~s}$, and no corresponding source was found within the XRT position to a $3 \sigma$ limit in the white filter of $\gtrsim 21.3 \mathrm{mag}$ using data over $\delta t=4400-6100 \mathrm{~s}$ (uncorrected for Galactic extinction; Breeveld \& Stamatikos 2011).

We obtained $i$-band observations with ACAM mounted on the $4.2 \mathrm{~m}$ William Herschel Telescope (WHT) at $\delta t=15.4 \mathrm{hr}$. In a total exposure time of $600 \mathrm{~s}$ (Table 2), we detect a single source within the enhanced XRT error circle with $i=22.77 \pm 0.29 \mathrm{mag}$, where the zero point has been determined using sources in common with the Sloan Digital Sky Survey (SDSS) catalog (Figure 6). To assess any fading associated with this source or within the XRT position, we obtained $i$-band imaging with LDSS3 starting on 2011 June 27.83 UT and no longer detect any source within the error circle to $i \gtrsim 24.7 \mathrm{mag}$, confirming that the source has faded by $\gtrsim 2 \mathrm{mag}$. Therefore, we consider this source to be the optical afterglow of GRB 110112A.

To determine the position of the afterglow, we perform absolute astrometry using 108 point sources in common with SDSS and calculate an astrometric tie rms of $00^{\prime} 11$. The resulting afterglow position is R.A. $=21^{\mathrm{h}} 59^{\mathrm{m}} 43^{\mathrm{s}} .85$ and decl. $=+26^{\circ} 27^{\prime} 23^{\prime \prime} .89$ (J2000) with a centroid uncertainty of $0^{\prime \prime} .09$ determined with Source Extractor, which, together with the astrometric tie uncertainty, gives a total positional uncertainty of $0{ }^{\prime \prime} 14$. We note that this source's position is not consistent with the $R=19.6 \pm 0.3$ source claimed by Xin et al. (2011). Furthermore, we do not detect any source at this position in any of our observations.

To perform a more thorough search for a coincident host galaxy, we obtained $r$-band observations with LDSS3 on 2011 June 27.83 UT and $i$-band observations with Gemini-North/ GMOS on 2011 July 28.46 UT. In these deeper observations, we do not detect any sources within the XRT error circle to limits of $r \gtrsim 25.5 \mathrm{mag}$ and $i \gtrsim 26.2 \mathrm{mag}$ (Table 2 ). We further assess the probability of potential host galaxies outside the XRT position in Section 4.3.

\section{AFTERGLOW PROPERTIES}

We utilize the X-ray and optical/NIR observations to constrain the explosion properties and circumburst environments of GRBs 100625A, 101219A, and 110112A. We adopt the standard synchrotron model for a relativistic blast wave in a constant density medium (interstellar medium), as expected for a nonmassive star progenitor (Sari et al. 1999; Granot \& Sari 2002). This model provides a mapping from the broadband afterglow flux densities to physical parameters: isotropic-equivalent kinetic energy $\left(E_{\mathrm{K} \text {,iso }}\right)$, circumburst density $\left(n_{0}\right)$, fractions of postshock energy in radiating electrons $\left(\epsilon_{e}\right)$ and magnetic fields $\left(\epsilon_{B}\right)$, and the electron power-law distribution index, $p$, with 


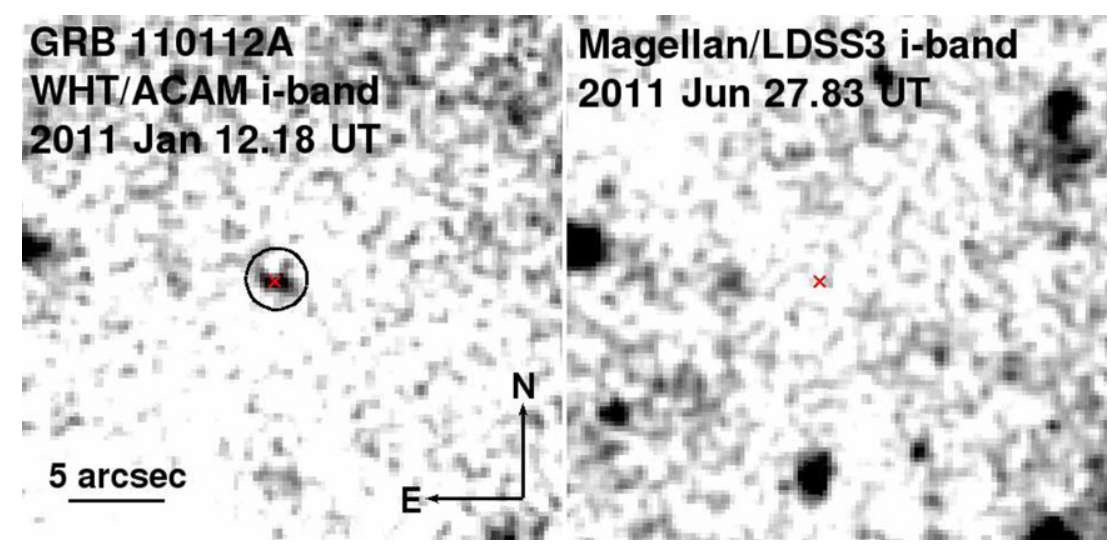

Figure 6. The optical afterglow of GRB 110112A. The XRT error circle has a radius of 1". 6 ( $90 \%$ containment; black) and the red cross marks the centroid of the optical afterglow, with a $1 \sigma$ uncertainty of 0 ' 14 (afterglow centroid + absolute tie to SDSS) and $i=22.77 \pm 0.29$. Left: WHT/ACAM $i$-band observations at $\delta t=0.64$ days. Right: Magellan/LDSS3 $i$-band observations at $\delta t=166$ days.

(A color version of this figure is available in the online journal.)

$N(\gamma) \propto \gamma^{-p}$ for $\gamma \gtrsim \gamma_{\min }$. Since we have optical and X-ray observations for these three bursts, we focus on constraining the location of the cooling frequency $\left(v_{c}\right)$ with respect to the X-ray band because it affects the afterglow flux dependence on $E_{\mathrm{K} \text {,iso }}$ and $n_{0}$. For each burst, we determine this by comparing the temporal $\left(\alpha_{X}\right)$ and spectral $\left(\beta_{X} \equiv 1-\Gamma\right)$ indices to the closure relation $\alpha-3 \beta / 2$ : for $p>2$, if $v_{c}>v_{X}, \alpha-3 \beta / 2=0$, while for $v_{c}<v_{X}, \alpha-3 \beta / 2=1 / 2$. We also infer the extinction, $A_{V}^{\text {host }}$, by a comparison of the optical and X-ray data.

\subsection{GRB 100625A}

From the X-ray light curve and spectrum of GRB 100625A, we measure a temporal decay index of $\alpha_{X}=-1.45 \pm 0.08$ and a spectral index of $\beta_{X}=-1.5 \pm 0.2$, which gives $\alpha_{X}-3 \beta_{X} / 2=0.79 \pm 0.34$. This indicates that $v_{c}<v_{X}$ and therefore $p=2.7 \pm 0.2$.

From our derived value of $N_{\mathrm{H}, \text { int }} \lesssim 1.7 \times 10^{21} \mathrm{~cm}^{-2}$, we infer $A_{V}^{\text {host }} \lesssim 0.8 \mathrm{mag}(3 \sigma)$ in the rest frame of the burst using the Galactic $N_{\mathrm{H}}$-to- $A_{V}$ conversion, $N_{\mathrm{H} \text {,int }} / A_{V} \approx 2.0 \times 10^{21}$ (Predehl \& Schmitt 1995; Watson 2011). We can also investigate the presence of extinction by comparing the X-ray flux and the optical upper limit at $\delta t \approx 0.5$ days. If we assume a maximum value of $v_{c, \text { max }} \approx 2.4 \times 10^{17} \mathrm{~Hz}(1 \mathrm{keV})$ and extrapolate the $\mathrm{X}$-ray flux density of $\approx 9 \times 10^{-3} \mu \mathrm{Jy}$ to the optical band using $\beta=-(p-1) / 2=-0.85$ to obtain the lowest bound on the expected afterglow flux in the absence of extinction, we estimate $F_{\nu, \text { opt }} \approx 0.24 \mu \mathrm{Jy}(i=25.4 \mathrm{mag})$. Given the observed limit of $F_{\nu, \mathrm{opt}} \lesssim 2.9 \mu \mathrm{Jy}(i \gtrsim 22.7 \mathrm{mag})$, this does not conflict with this lower bound, and the afterglow observations are consistent with no extinction.

We can therefore use the X-ray data and optical afterglow limits to constrain $E_{\mathrm{K} \text {,iso }}$ and $n_{0}$. Assuming that the X-ray flux is from the forward shock, we can directly obtain $E_{\mathrm{K} \text {,iso }}$ by (Granot \& Sari 2002)

$$
E_{\mathrm{K}, \mathrm{iso}, 52}^{4.7 / 4} \epsilon_{e,-1}^{1.7} \epsilon_{B,-1}^{0.7 / 4} \approx 5.7 \times 10^{-3}
$$

where $E_{\mathrm{K}, \text { iso }, 52}$ is in units of $10^{52} \mathrm{erg}$, and $\epsilon_{e}$ and $\epsilon_{B}$ are in units of $10^{-1}$, and we have used $z=0.452$. The $X$-ray flux density at $\delta t \approx 10^{4} \mathrm{~s}$ is $F_{v, X} \approx 9.1 \times 10^{-3} \mu \mathrm{Jy}(1 \mathrm{keV})$, and therefore $E_{\mathrm{K} \text {,iso }} \approx 1.2 \times 10^{50} \mathrm{erg}\left(\epsilon_{e}=\epsilon_{B}=0.1\right)$. At $z=0.452, E_{\gamma \text {,iso }} \approx 4.3 \times 10^{50} \mathrm{erg}(20-2000 \mathrm{keV}$ from the Konus-Wind fluence), which gives a $\gamma$-ray efficiency of $\eta_{\gamma} \approx 0.8$. If we instead assume $\epsilon_{e}=0.1$ and $\epsilon_{B}=0.01$, then $E_{\mathrm{K}, \text { iso }} \approx 1.7 \times 10^{50} \mathrm{erg}$, and $\eta_{\gamma} \approx 0.7$.

For $v_{m}<v_{\text {opt }}<v_{c}$ (where $v_{m}$ is the synchrotron peak frequency), the optical afterglow brightness depends on a combination of $E_{\mathrm{K} \text {,iso }}$ and $n_{0}$. Therefore, the riz-band observed limits on the afterglow translate to an upper limit on the physical parameters, given by

$$
E_{\mathrm{K}, \text { iso }, 52}^{5.7 / 4} n_{0}^{0.5} \epsilon_{e,-1}^{1.7} \epsilon_{B,-1}^{3.7 / 4} \lesssim 2.5 \times 10^{-3},
$$

where $n_{0}$ is in units of $\mathrm{cm}^{-3}$. Assuming $\epsilon_{e}=\epsilon_{B}=0.1$ and using $E_{\mathrm{K} \text {,iso }}=1.2 \times 10^{50} \mathrm{erg}$, we obtain $n \lesssim 1.5 \mathrm{~cm}^{-3}$. If we instead assume $\epsilon_{e}=0.1$ and $\epsilon_{B}=0.01$, then $n_{0} \lesssim 40 \mathrm{~cm}^{-3}$. For both scenarios, we obtain $v_{c} \gtrsim 4 \times 10^{15} \mathrm{~Hz}(\gtrsim 0.02 \mathrm{keV})$, consistent with our assumption that $v_{c}<v_{X}$.

\subsection{GRB 101219A}

From the X-ray light curve and spectrum, we measure $\alpha_{X}=-1.37 \pm 0.13$ and $\beta_{X}=-0.8 \pm 0.1$, which gives $\alpha_{X}-3 \beta_{X} / 2=0.17 \pm 0.23$, suggesting that $v_{c}>v_{X}$. The resulting value of $p$ is $2.7 \pm 0.1$. We note that the closure relation is consistent with the alternative scenario for $>2 \sigma$.

Since the optical afterglow flux may be subject to an appreciable amount of extinction, as suggested by the intrinsic absorption in the X-ray spectrum (Section 2.2.1), the most reliable proxy for $E_{\mathrm{K} \text {,iso }}$ and $n_{0}$ is the X-ray afterglow flux. Using the last XRT data point at $\delta t \approx 7 \times 10^{3} \mathrm{~s}$, which has $F_{v, X} \approx 0.03 \mu \mathrm{Jy}(1 \mathrm{keV})$, we infer the following relationship between $E_{\mathrm{K} \text {,iso }}$ and $n_{0}$ :

$$
E_{\mathrm{K}, \text { iso }, 52}^{5.7 / 4} n_{0}^{0.5} \epsilon_{e,-1}^{1.7} \epsilon_{B,-1}^{3.7 / 4} \approx 1.3 \times 10^{-3},
$$

where we have used $z=0.718$. At this redshift, we find $E_{\gamma \text {,iso }} \approx$ $4.8 \times 10^{51}$ erg (20-10 $\mathrm{keV}$ using the Konus-Wind fluence). Assuming $E_{\gamma \text {,iso }} \approx E_{\mathrm{K} \text {,iso }}$, we infer $n_{0} \approx 1.3 \times 10^{-5} \mathrm{~cm}^{-3}$ for $\epsilon_{e}=\epsilon_{B}=0.1$. With these values, $v_{c} \approx 6 \times 10^{19} \mathrm{~Hz}$ $(250 \mathrm{keV})$, consistent with our assumption that $v_{c}>v_{X}$. We note that this assumption is violated for $n_{0} \gtrsim 4 \times 10^{-3} \mathrm{~cm}^{-3}$. If instead we use $\epsilon_{e}=0.1$ and $\epsilon_{B}=0.01$, then we obtain $n_{0} \approx 9 \times 10^{-4} \mathrm{~cm}^{-3}$ and $v_{c} \approx 2 \times 10^{19} \mathrm{~Hz}(80 \mathrm{keV})$, which is again self-consistent, and find this assumption is violated for $n_{0} \gtrsim 0.1 \mathrm{~cm}^{-3}$. Therefore, the X-ray data suggest an explosion environment with $n_{0} \approx 10^{-5}-10^{-3} \mathrm{~cm}^{-3}$ for GRB 101219A.

We investigate the presence of extinction intrinsic to the host galaxy by comparing the X-ray and NIR observations, since the 
NIR data provide a stronger constraint than the optical band. Since the X-ray and NIR bands lie on the same segment of the synchrotron spectrum, the spectral slope is given by $\beta_{\mathrm{NIR}-\mathrm{X}}=$ $\beta_{X} \approx-0.8$. At the time of our first $J$-band observations at $\delta t \approx$ $1 \mathrm{hr}$, the X-ray flux density is $0.06 \mu \mathrm{Jy}$, leading to an expected $J$-band flux density of $F_{v, J} \approx 14.7 \mu \mathrm{Jy}(21 \mathrm{mag})$. This is above the limit of our observations, $\lesssim 1.4 \mu \mathrm{Jy}$ ( $\gtrsim 23.6 \mathrm{mag}$ ), indicating that $A_{J} \gtrsim 2.5 \mathrm{mag}$. Using a Milky Way extinction curve (Cardelli et al. 1989), this indicates that $A_{V}^{\text {host }} \gtrsim 4.2$ mag in the rest frame of the burst. In addition, using the Galactic relation between $N_{\mathrm{H}}$ and $A_{V}$, this implies $N_{\mathrm{H} \text {,int }} \gtrsim 7.5 \times 10^{21} \mathrm{~cm}^{-2}$, which does not necessarily violate our inferred value from the X-ray spectrum of $N_{\mathrm{H} \text {,int }}=(6.6 \pm 2.0) \times 10^{21} \mathrm{~cm}^{-2}$. Therefore, the broadband afterglow spectrum requires an appreciable amount of extinction.

\subsection{GRB 110112A}

From the $X$-ray light curve and spectrum, we measure $\alpha_{X}=-1.10 \pm 0.05$ and $\beta_{X}=-1.2 \pm 0.2$, giving $\alpha_{X}-3 / 2 \beta_{X}=$ $0.70 \pm 0.30$ indicating $v_{c}<v_{X}$. The resulting value of $p$ is $2.1 \pm 0.1$.

From our derived value of $N_{\mathrm{H} \text {,int }} \lesssim 1.6 \times 10^{21} \mathrm{~cm}^{-2}$, we infer $A_{V}^{\text {host }} \lesssim 0.9$ mag in the rest frame of the burst using the Galactic relation. We can measure the cooling frequency by comparing the $\mathrm{X}$-ray and optical fluxes at $\delta t \approx 0.64$ days. At this time, $F_{v, X} \approx 6.6 \times 10^{-3} \mu \mathrm{Jy}$ and $F_{v, \mathrm{opt}} \approx 2.8 \mu \mathrm{Jy}$. Using $p=2.1$ and the location of the optical and $\mathrm{X}$-ray bands, we then estimate that $v_{c} \approx 1.6 \times 10^{15} \mathrm{~Hz}\left(\approx 7 \times 10^{-3} \mathrm{keV}\right)$, which agrees with our assumption that $v_{c}<v_{X}$. The cooling frequency is dependent on a combination of physical parameters and gives the constraint

$$
E_{\mathrm{K}, \text { iso, }, 52}^{-0.5} n_{0}^{-1} \epsilon_{B,-1}^{-1.5} \approx 5.4
$$

where we have assumed a fiducial redshift of $z=0.5$, the median of the observed short GRB population. We then use the $\mathrm{X}$-ray afterglow flux at $\delta t \approx 0.64$ days to determine $E_{\mathrm{K} \text {,iso }}$ by

$$
E_{\mathrm{K}, \text { iso }, 52}^{4.1 / 4} \epsilon_{e,-1}^{1.1} \epsilon_{B,-1}^{0.1 / 4} \approx 0.023 .
$$

Our final constraint comes from the optical afterglow brightness, given by

$$
E_{\mathrm{K}, \mathrm{iso}, 52}^{5.1 / 4} n_{0}^{0.5} \epsilon_{e,-1}^{1.1} \epsilon_{B,-1}^{3.1 / 4} \approx 0.01
$$

Assuming $\epsilon_{e}=0.1$ and $z=0.5$, we obtain the solution $E_{\mathrm{K}, \text { iso }} \approx 2.5 \times 10^{50} \mathrm{erg}, n_{0} \approx 1.5 \mathrm{~cm}^{-3}$, and $\epsilon_{B} \approx 0.08$. At this redshift, $E_{\gamma \text {,iso }} \approx 9.5 \times 10^{49} \mathrm{erg}$ (determined from the Swift fluence and applying a correction factor of five to represent $\approx 1-10^{4} \mathrm{keV}$ ). If we consider a high-redshift origin for GRB $110112 \mathrm{~A}$ of $z=2$, then we infer larger energies of $E_{\mathrm{K} \text {,iso }} \approx 3.6 \times 10^{51} \mathrm{erg}$ and $E_{\gamma \text {,iso }} \approx 1.5 \times 10^{51} \mathrm{erg}$, a lower value of $\epsilon_{B} \approx 0.01$, and a lower density, $n_{0} \approx 0.18 \mathrm{~cm}^{-3}$. In both cases, $\eta_{\gamma} \approx 0.3$.

\section{HOST GALAXY PROPERTIES}

\subsection{GRB 100625A}

The XRT position of GRB 100625A fully encompasses a single galaxy, which we call G1 (Figure 2). To assess the probability that the burst originated from G1, we calculate the probability of chance coincidence, $P_{\mathrm{cc}}(<\delta R)$, at a given angular separation $(\delta R)$, and apparent magnitude $(m)$ for galaxies within $15^{\prime}$ (the field of view of our images) of the burst position (Bloom et al. 2002; Berger 2010a). For G1, we conservatively assume

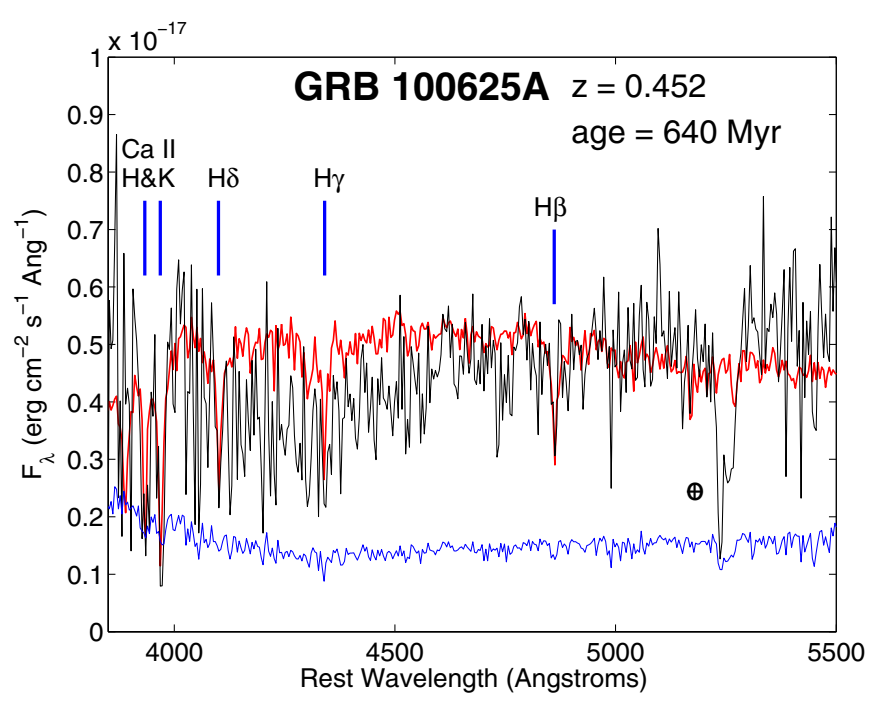

Figure 7. LDSS3 spectrum of the early-type host galaxy of GRB 100625A, binned with a 3-pixel boxcar (black: data; blue: error spectrum). Also shown is the best-fit SSP template (red; Bruzual \& Charlot 2003) with a stellar population age of $640 \mathrm{Myr}$ at a redshift of $z=0.452 \pm 0.002$. Fits are performed on the unbinned data. The locations of the Balmer absorption lines and $\mathrm{Ca}$ II $\mathrm{H} \& \mathrm{~K}$ are labeled.

(A color version of this figure is available in the online journal.)

$\delta R=3 \sigma_{\mathrm{XRT}} \approx 3 . .4$ and calculate $P_{\mathrm{cc}}(<\delta R) \approx 0.04$. The remaining bright galaxies in the field have substantially higher values of $P_{\mathrm{cc}}(<\delta R) \gtrsim 0.17$, and a search for galaxies within $5^{\circ}$ of the GRB position using the NASA/IPAC Extragalactic Database (NED) yields only objects with $P_{\mathrm{cc}} \gtrsim 0.98$. From these probabilistic arguments, we consider G1 to be the host galaxy of GRB 100625A.

To determine the host galaxy's redshift, we fit the LDSS3 spectrum over the wavelength range of $5200-8000 \AA$ with simple stellar population (SSP) spectral evolution models at fixed ages ( $\tau=0.29,0.64,0.90,1.4$, and $2.5 \mathrm{Gyr}$ ) provided as part of the GALAXEV library (Bruzual \& Charlot 2003); at wavelengths outside this range, the signal-to-noise is too low to contribute significantly to the fit. We use $\chi^{2}$-minimization with redshift as the single free parameter and perform the fit on the unbinned data. The resulting best-fit redshift is $z=0.452 \pm 0.002\left(\chi_{v}^{2}=1.3\right.$ for $\left.1861 \mathrm{dof}\right)$, determined primarily by the location of the $4000 \AA$ break and the main absorption features of $\mathrm{Ca} I \mathrm{H} \& \mathrm{~K}, \mathrm{H} \beta, \mathrm{H} \gamma$, and $\mathrm{H} \delta$. The shape of the break is best fit by the template with $\tau=0.64 \mathrm{Gyr}$ (Figure 7$)$, and poorer fits $\left(\chi_{v}^{2} \gtrsim 2\right)$ are found for SSPs with younger or older ages. Due to the strength of the $4000 \AA$ break, deep absorption features, lack of emission lines, and old age, we classify this host as an early-type galaxy. We note that the shape of the spectrum does not require any intrinsic extinction, which is consistent with the results from the afterglow observations.

We do not find an emission feature corresponding to $\left[\mathrm{O}_{\text {II }}\right] \lambda 3727$. Using the error spectrum, we calculate the expected integrated flux for a $3 \sigma$ emission doublet centered at $\lambda=3727 \AA$ with a width of $\approx 10 \AA$. We find an expected upper limit of $F_{[\mathrm{OII}} \lesssim 4.3 \times 10^{-17} \mathrm{erg} \mathrm{cm}^{-2} \mathrm{~s}^{-1}$, which translates to $L_{\text {[О II] }} \lesssim 2.2 \times 10^{40} \mathrm{erg} \mathrm{s}^{-1}$ at the redshift of the burst. Using the standard relation, $\mathrm{SFR}=(1.4 \pm 0.4) M_{\odot} \mathrm{yr}^{-1} L_{[\mathrm{O} \mathrm{II}], 41}$ (Kennicutt 1998), we derive a $3 \sigma$ upper limit of SFR $\lesssim$ $0.3 M_{\odot} \mathrm{yr}^{-1}$ for the host galaxy.

We use the grizJK $K_{s}$-band photometry to infer the stellar population age and mass of the host galaxy with the Maraston (2005) 


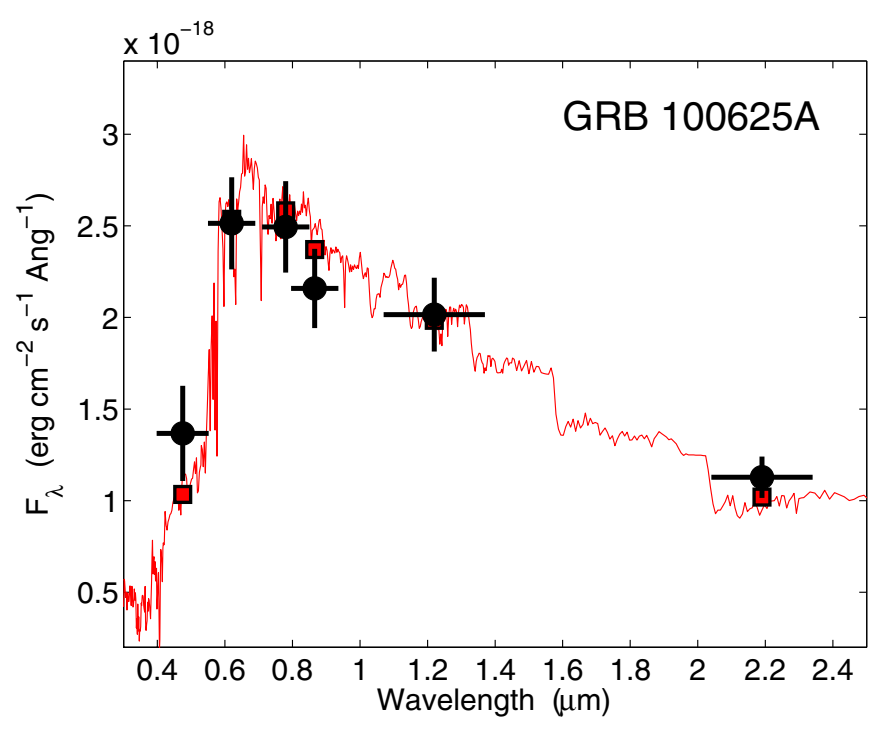

Figure 8. grizJ $_{s}$ photometry for the host galaxy of GRB 100625A (black circles). The best-fit model (red squares and line; Maraston 2005) is characterized by $\tau \approx 0.8$ Gyr and $M_{*} \approx 4.6 \times 10^{9} M_{\odot}$.

(A color version of this figure is available in the online journal.)

evolutionary stellar population synthesis models, employing a Salpeter initial mass function and a red giant branch morphology. We fix $A_{V}^{\text {host }}=0 \mathrm{mag}$ as inferred from the absence of $N_{\mathrm{H}, \text { int }}$ (Section 2.1.1), $z=0.452$ as inferred from the spectrum, and metallicity $Z=Z_{\odot}$, and allow the stellar population age $(\tau)$ and stellar mass $\left(M_{*}\right)$ to vary. The resulting best-fit model is characterized by $\tau \approx 0.8 \mathrm{Gyr}$, in good agreement with the fit to the spectrum, and $M_{*} \approx 4.6 \times 10^{9} M_{\odot}$. The model and broadband photometry are shown in Figure 8.

\subsection{GRB 101219A}

The XRT position of GRB 101219A fully encompasses a single galaxy (G1; Figure 4). We perform the same probability of chance coincidence analysis described in Section 4.1 using $\delta R=3 \sigma_{\mathrm{XRT}}$ and find $P_{\mathrm{cc}}(<\delta R) \approx 0.06$ for $\mathrm{G} 1$, while the remaining bright galaxies within $5^{\prime}$ of the burst have $P_{\mathrm{cc}}(<\delta R) \gtrsim 0.23$. Furthermore, a search within $5^{\circ}$ of the position with NED yields only galaxies with $P_{\mathrm{cc}}(<\delta R) \approx 1$. We therefore consider G1 to be the most probable host galaxy of GRB 101219A.

We examine the host spectrum of GRB 101219A to determine the redshift and physical characteristics of the stellar population. We identify two emission features in the co-added spectrum at $\lambda_{\text {obs }}=6401.65 \AA$ and $\lambda_{\text {obs }}=8599.50 \AA$ that are also present in the individual two-dimensional spectra prior to co-addition. If these features correspond to $[\mathrm{O} \mathrm{II}] \lambda 3727$ and $[\mathrm{O} \mathrm{III}] \lambda 5007$, their locations give a common redshift of $z=0.718$. Furthermore, we do not find a common redshift solution for an alternative set of features, so we consider the host galaxy to be at $z=0.718$. In addition, we note the presence of marginal emission features at the expected locations of $\mathrm{H} \beta$ and [O III] $\lambda 4959$; however, these locations are contaminated by sky line residuals. Finally, we detect absorption at the locations of $\mathrm{H} \varepsilon$ and $\mathrm{H} \delta$ (Figure 9).

To determine the age and host extinction, we use stellar population spectral templates with fixed ages of $\tau=5,25,100$, and 290 Myr (Bruzual \& Charlot 2003) to fit the continuum; ages outside this range do not fit the overall shape of the spectrum. We apply corrections for both Galactic extinction $\left(A_{V}=0.16 \mathrm{mag}\right.$ at $z=0$; Schlafly \& Finkbeiner 2011)

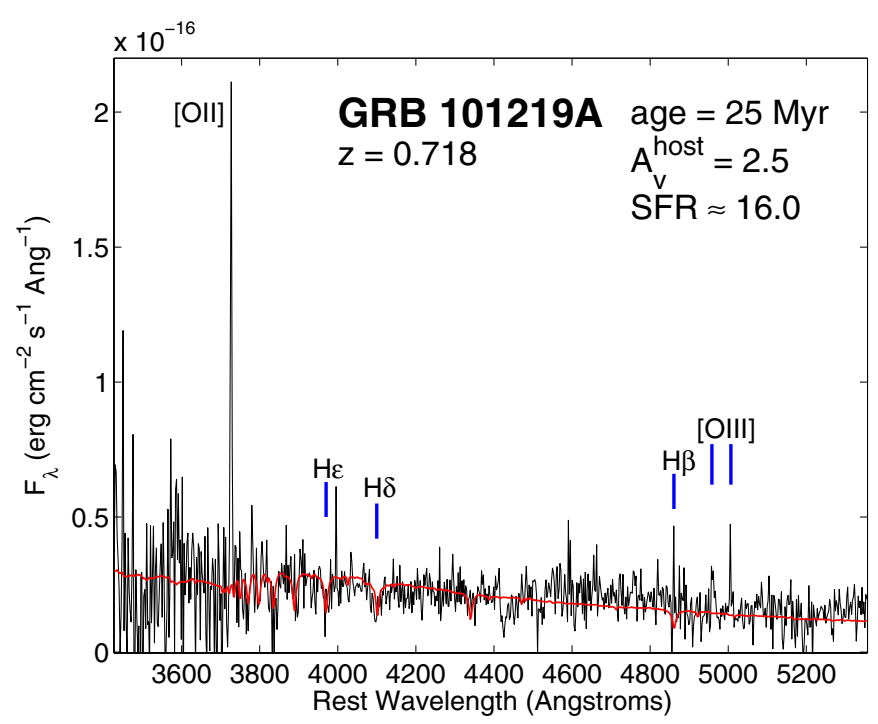

Figure 9. GMOS-N spectrum of the host galaxy of GRB 101219A, binned with a 3-pixel boxcar (black). The spectrum is corrected for Galactic extinction and $A_{V}^{\text {host }}=2.5 \mathrm{mag}$. The stellar population model has $\tau=25 \mathrm{Myr}$ (red; Bruzual $\&$ Charlot 2003). The [O II] $\lambda 3727$ and [O III] $\lambda 5007$ emission features are at a common redshift of $z=0.718$. Also labeled are the locations of the Balmer lines $\mathrm{H} \epsilon$ and $\mathrm{H} \delta$, and marginal emission features at $\mathrm{H} \beta$ and the [O III] doublet. From $\left[\mathrm{O}_{\mathrm{II}}\right] \lambda 3727$ we deduce $\mathrm{SFR}=16.0 \pm 4.6 M_{\odot} \mathrm{yr}^{-1}$. (Kennicutt 1998).

(A color version of this figure is available in the online journal.)

and $A_{V}^{\text {host }}$ at $z=0.718$ using a Milky Way extinction curve (Cardelli et al. 1989). The spectrum is best matched with the $\tau=25$ Myr template and $A_{V}^{\text {host }}=2.5$ mag. Since there is some degeneracy between age and $A_{V}^{\text {host }}$, imposing an older stellar population of $\tau=100 \mathrm{Myr}$ also provides a reasonable match, but requires a smaller amount of extinction of $A_{V}^{\text {host }} \approx 2$ mag. Older spectral templates predict a large break at $4000 \AA$ not seen in the spectrum, while younger templates lack the observed absorption lines. Therefore, a likely range of ages for the host galaxy is $\tau \approx 25-100$ Myr. Given the emission features and relatively young age, we classify this galaxy as late-type. The de-reddened spectrum for GRB 101219A, along with the $25 \mathrm{Myr}$ model, is shown in Figure 9.

From the extinction-corrected flux of $[\mathrm{O}$ II $] \lambda 3727, F_{[\mathrm{O}}{ }_{\text {II }} \approx$

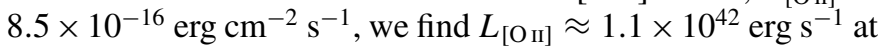
the redshift of the burst. Using the standard relation (Kennicutt 1998), we derive an SFR of $16.0 \pm 4.6 M_{\odot} \mathrm{yr}^{-1}$.

We use the same procedure described in Section 4.1 to model the SED of the host galaxy to infer $\tau$ and $M_{*}$. We fix $z=0.718$ as inferred from the spectrum, $Z=Z_{\odot}$, and allow $\tau, M_{*}$, and $A_{V}^{\text {host }}$ to vary. The resulting best-fit model is characterized by $A_{V}^{\text {host }} \approx 1.5 \mathrm{mag}, \tau \approx 15-25 \mathrm{Myr}$, and $M_{*} \approx 1.4 \times 10^{9} M_{\odot}$, which is consistent with the parameters derived from the spectrum and afterglow. The broadband photometry and best-fit stellar population model are shown in Figure 10.

\subsection{GRB 110112A}

For GRB 110112A, we do not detect a source in coincidence with the optical afterglow position or within the XRT error circle to a $3 \sigma$ limit of $i \gtrsim 26.2$ mag in our GMOS-N image (Figure 11). To determine which sources in the field are probable hosts, we calculate $P_{\mathrm{cc}}(<\delta R)$ for 15 galaxies within $\sim 3^{\prime}$ of the GRB position, the field of view of our GMOS-N $i$-band image. These galaxies were selected by discarding noticeably fainter galaxies with increasing $\delta R$ since these objects will have $P_{\mathrm{cc}}(<\delta R) \sim 1$. 


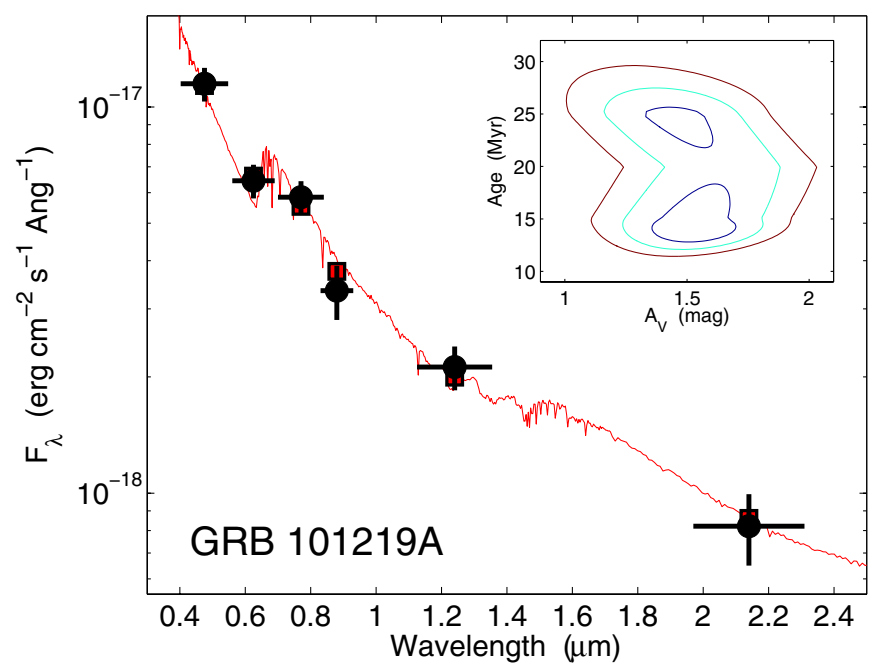

Figure 10. grizJK $_{s}$-band photometry of the host galaxy of GRB 101219A (black circles). The best-fit model (red squares and line; Maraston 2005) is characterized by $A_{V}^{\text {host }} \approx 1.5 \mathrm{mag}, \tau \approx 15-25 \mathrm{Myr}$, and $M_{*} \approx 1.4 \times 10^{9} M_{\odot}$. The age $-A_{V}^{\text {host }}$ contours of $1 \sigma$ (blue), $2 \sigma$ (cyan), and $3 \sigma$ (red) solutions are shown in the inset.

(A color version of this figure is available in the online journal.)

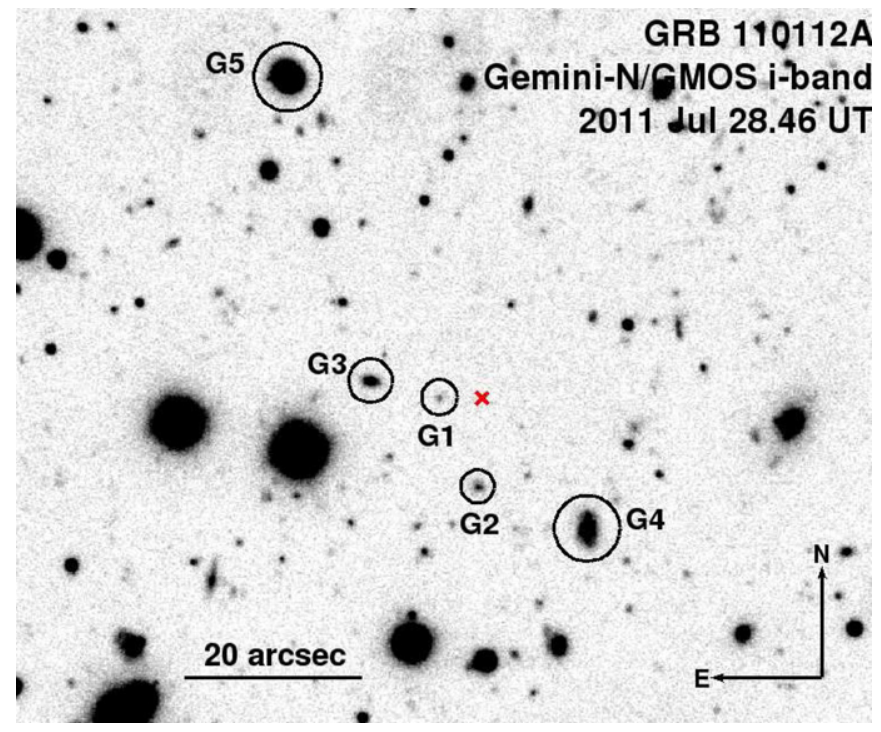

Figure 11. Left: Gemini-N/GMOS $i$-band observations of the field of GRB 110112A on $2011 \mathrm{Jul} 28.46$ UT. The position of the optical afterglow is marked by the red cross. The five galaxies with the lowest probabilities of chance coincidence are circled and labeled G1-G5. The galaxy with the lowest value of $P(<\delta R)$ is $\mathrm{G} 1$, located 4."8 from the optical afterglow position.

(A color version of this figure is available in the online journal.)

We find that nine of these galaxies have $P_{\mathrm{cc}}(<\delta R) \lesssim 0.85$ (Figure 12). The two most probable host galaxies, G1 and G4 (Figures 11 and 12), have $P_{\mathrm{cc}}(<\delta R)=0.43$ and 0.54 , respectively, and offsets of $\delta R=44^{\prime \prime} 8$ and 11". 1 . In addition, we search for bright galaxies within $5^{\circ}$ of the GRB position using $\mathrm{NED}$, but all additional cataloged galaxies have $P_{\mathrm{cc}}(<\delta R) \gtrsim$ 0.98 . Given the relatively high values for $P_{\mathrm{cc}}(<\delta R)$, we do not find a convincing putative host for GRB 110112A.

It is also plausible that GRB 110112A originated from a galaxy fainter than the detection threshold of our observations. For instance, a $\approx 27$ mag host would require $\delta R \lesssim 22^{\prime \prime} 0$ while a $\approx 28$ mag host would require $\delta R \lesssim 1^{\prime \prime} 3$, to be a more probable host than G1. However, to be a $27-28$ mag galaxy convincing

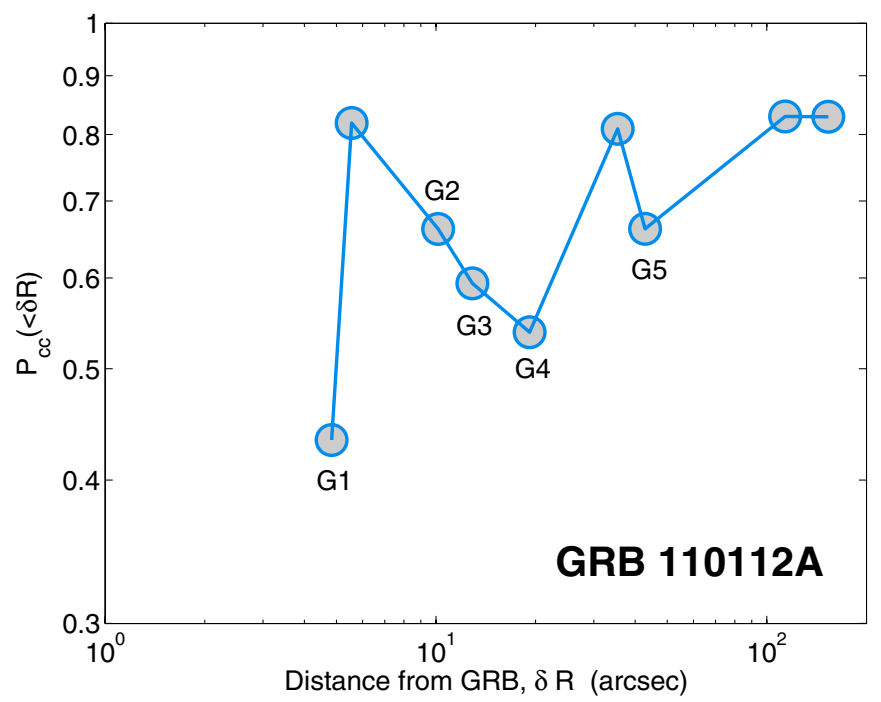

Figure 12. Probability of chance coincidence, $P_{\mathrm{cc}}(<\delta R)$, as a function of angular distance from the optical afterglow position of GRB 110112A. There are nine galaxies in the $2^{\prime}$ field with $P_{\mathrm{cc}}(<\delta R)<0.85$. The five galaxies with the lowest $P(<\delta R)$ are labeled G1-G5. The galaxy G1 has the lowest probability of chance coincidence $P(<\delta R)=0.43$.

(A color version of this figure is available in the online journal.)

enough to make a host association $\left(P_{\mathrm{cc}}(<\delta R) \lesssim 0.05\right)$ would require a smaller offset of $\delta R \lesssim 0 ! 5$. We note that the lack of potential host is in contrast to previous "host-less" short GRBs (Berger 2010a). The high inferred density due to the bright optical afterglow (Section 3) is suggestive of a high-redshift origin as opposed to a progenitor system that was kicked outside of its host galaxy.

\section{STELLAR POPULATION CHARACTERISTICS}

Of the 30 short GRBs with host associations $\left(P_{\mathrm{cc}} \lesssim 0.05\right.$; Table 3), GRB 100625A is the fifth short GRB associated with a spectroscopically confirmed early-type host galaxy (Gehrels et al. 2005; Berger et al. 2005; Bloom et al. 2006, 2007; Fong et al. 2011), near the median redshift of the short GRB population (Figure 13). In contrast, GRB 101219A is associated with a $z=0.718$ late-type galaxy that is actively star-forming with characteristics similar to the majority of the short GRB late-type host population (Berger 2009). Finally, GRB 110112A joins a growing number of short GRBs with subarcsecond positions but no obvious coincident host galaxy to deep limits of $\gtrsim 26$ mag (Berger 2010a), although unlike previous events, the case for a large offset is less clear.

Short GRBs with subarcsecond positions and coincident hosts have a median projected physical offset of $\sim 5 \mathrm{kpc}$ (Fong et al. 2010), which, in the context of an NS-NS/NS-BH progenitor, can be interpreted as the result of natal kicks and moderate delay times (Fryer et al. 1999; Belczynski et al. 2006). At the inferred redshifts of GRBs 100625A and 101219A, the upper limits on the projected physical offsets set by the radii of the X-ray positions are $\lesssim 10.3$ and $\lesssim 12.3 \mathrm{kpc}$, respectively, which agree with the observed offset distribution. Assuming a fiducial redshift of $z=0.5$, GRB 110112A would be located $29 \pm 3 \mathrm{kpc}$ away from the closest and most probable host galaxy, but this association is much less definitive $\left(P_{\mathrm{cc}}(<\delta R) \approx 0.43\right)$ than previous host-less bursts (Berger 2010a). Imaging with the Hubble Space Telescope may enable the detection of a faint coincident host. These offsets are in contrast to long 
Table 3

Short GRB Host Galaxy Morphologies

\begin{tabular}{|c|c|c|c|c|c|c|}
\hline GRB & $\begin{array}{c}T_{90}{ }^{\mathrm{a}} \\
(\mathrm{s})\end{array}$ & $z^{\mathrm{b}}$ & Type $^{c}$ & $\begin{array}{l}\text { 90\% XRT Uncert. } \\
\text { (arcsec) }\end{array}$ & $P_{\mathrm{cc}}(<\delta R)$ & References \\
\hline \multicolumn{7}{|c|}{ Subarcsecond localized } \\
\hline 050709 & $0.07 / 130$ & 0.161 & $\mathrm{~L}$ & & $3 \times 10^{-3}$ & $1-3$ \\
\hline 050724A & 3 & 0.257 & $\mathrm{E}$ & & $2 \times 10^{-5}$ & $4-5$ \\
\hline 051221A & 1.4 & 0.546 & $\mathrm{~L}$ & & $5 \times 10^{-5}$ & $6-7$ \\
\hline 060121 & 2.0 & $<4.1$ & $?$ & & $2 \times 10^{-3}$ & $8-9$ \\
\hline 060313 & 0.7 & $<1.7$ & $?$ & & $3 \times 10^{-3}$ & $10-11$ \\
\hline 061006 & $0.4 / 130$ & 0.4377 & $\mathrm{~L}$ & & $4 \times 10^{-4}$ & $12-15$ \\
\hline 061201 & 0.8 & 0.111 & $\mathrm{H} / \mathrm{L}$ & & $\cdots / 0.08$ & $9,16-17$ \\
\hline 070429B & 0.5 & 0.9023 & $\mathrm{~L}$ & & $3 \times 10^{-3}$ & $18-19$ \\
\hline 070707 & 1.1 & $<3.6$ & $?$ & & $7 \times 10^{-3}$ & $20-21$ \\
\hline 070714B & $2.0 / 64$ & 0.9224 & $\mathrm{~L}$ & & $5 \times 10^{-3}$ & $19,22-23$ \\
\hline 070724A & 0.4 & 0.457 & $\mathrm{~L}$ & & $8 \times 10^{-4}$ & $24-25$ \\
\hline 070809 & 1.3 & 0.473 & $\mathrm{H} / \mathrm{E}$ & & $\cdots / 0.03$ & 9,26 \\
\hline 071227 & $1.8^{\mathrm{e}}$ & 0.381 & $\mathrm{~L}$ & & 0.01 & $27-29$ \\
\hline 080503 & $0.3 / 170$ & $<4.2$ & $\mathrm{H} / ?$ & & $\cdots / 0.1$ & $9,30-31$ \\
\hline 080905A & 1.0 & 0.1218 & $\mathrm{~L}$ & & 0.01 & $32-33$ \\
\hline 081226A & 0.4 & $<4.1$ & $?$ & & 0.01 & $34-35$ \\
\hline 090305 & 0.4 & $<4.1$ & $\mathrm{H} / ?$ & & $\cdots / 0.06$ & 9,36 \\
\hline 090426A & 1.3 & 2.609 & $\mathrm{~L}$ & & $1.5 \times 10^{-4}$ & $37-38$ \\
\hline 090510 & 0.3 & 0.903 & $\mathrm{~L}$ & & $8 \times 10^{-3}$ & $39-40$ \\
\hline 090515 & 0.04 & 0.403 & $\mathrm{H} / \mathrm{E}$ & & $\cdots / 0.15$ & 9,41 \\
\hline 091109B & 0.3 & $<4.4$ & $?$ & & $\ldots$ & $42-43$ \\
\hline 100117A & 0.3 & 0.915 & $\mathrm{E}$ & & $7 \times 10^{-5}$ & $44-45$ \\
\hline $110112 \mathrm{~A}$ & 0.5 & $<5.3$ & $\mathrm{H} / ?$ & & 0.43 & 46 , This work \\
\hline $111020 \mathrm{~A}^{\mathrm{f}}$ & 0.4 & $\ldots$ & $?$ & & 0.01 & $47-48$ \\
\hline $111117 \mathrm{~A}^{\mathrm{fg}}$ & 0.5 & 1.3 & $\mathrm{~L}$ & & 0.02 & $49-50$ \\
\hline \multicolumn{7}{|c|}{ XRT only } \\
\hline 050509B & 0.04 & 0.225 & E & 3.8 & $5 \times 10^{-3}$ & $51-52$ \\
\hline $050813^{\mathrm{h}}$ & 0.6 & $0.72 / 1.8$ & $\mathrm{E} / ?$ & 2.9 & $\ldots$ & $53-57$ \\
\hline 051210 & 1.3 & $>1.4$ & $?$ & 1.6 & 0.04 & 14,58 \\
\hline 060502B & 0.09 & 0.287 & $\mathrm{E}$ & 5.2 & 0.03 & $59-60$ \\
\hline 060801 & 0.5 & 1.130 & $\mathrm{~L}$ & 1.5 & 0.02 & $61-62$ \\
\hline 061210 & $0.2 / 85$ & 0.4095 & $\mathrm{~L}$ & 3.9 & 0.02 & 14,63 \\
\hline 061217 & 0.2 & 0.827 & $\mathrm{~L}$ & 5.5 & $0.24^{\mathrm{i}}$ & 14,64 \\
\hline $070729^{\mathrm{g}}$ & 0.9 & 0.8 & $\mathrm{E}$ & 2.5 & 0.05 & $65-66$ \\
\hline 080123 & $0.4 / 115$ & 0.495 & $\mathrm{~L}$ & 1.7 & 0.004 & $67-68$ \\
\hline $100206 \mathrm{~A}$ & 0.1 & 0.4075 & $\mathrm{~L}$ & 3.3 & 0.02 & 69-70 \\
\hline $100625 \mathrm{~A}$ & 0.3 & 0.452 & $\mathrm{E}$ & 1.8 & 0.04 & 71 , This work \\
\hline 101219A & 0.6 & 0.718 & $\mathrm{~L}$ & 1.7 & 0.06 & 72 , This work \\
\hline
\end{tabular}

Notes.

a Swift 15-150 keV. For bursts with extended emission, both the duration of the prompt spike and the duration including extended emission are reported.

${ }^{\mathrm{b}}$ Upper limits on redshift are based on the detection of the UV/optical afterglow and therefore the lack of suppression blueward of the Lyman limit $\left(\lambda_{0}=912 \AA\right)$ or Ly $\alpha$ line $\left(\lambda_{0}=1216 \AA\right)$.

${ }^{\mathrm{c}} \mathrm{L}=$ late-type, $\mathrm{E}=$ early-type, ? = inconclusive type, $\mathrm{H}=$ "host-less." For each host-less burst, we also list the type of the galaxy with the lowest $P_{\mathrm{cc}}$ (Berger 2010a and this work).

d Only listed for XRT bursts (Goad et al. 2007; Evans et al. 2009).

e Evidence at the $4 \sigma$ level for extended emission is reported to $\delta t \approx 100 \mathrm{~s}$.

${ }^{\mathrm{f}}$ Bursts with no optical afterglow, localized by Chandra.

g Bursts with galaxy type classifications based on extensive broadband photometry (Leibler \& Berger 2010; Margutti et al. 2012). In particular, the host of GRB 070729 has an inferred age $(\approx 0.98 \mathrm{Gyr})$ and stellar mass $\left(\approx 4 \times 10^{10} M_{\odot}\right.$; Leibler \& Berger 2010) more consistent with an early-type designation.

${ }^{\mathrm{h}}$ There exists disagreement in the literature regarding the association of GRB 050813 with an early-type cluster galaxy at $z=0.72$ (Berger 2005; Foley et al. 2005; Prochaska et al. 2006) or a high-redshift cluster at $z=1.8$ (Berger 2006); thus, we only display this burst for completeness but do not include it in our demographics.

${ }^{\mathrm{i}}$ Despite the relatively high $P_{\mathrm{cc}}$, all surrounding galaxies have $P_{\mathrm{cc}}$ of order unity (Berger et al. 2007).

References. (1) Villasenor et al. 2005; (2) Fox et al. 2005; (3) Hjorth et al. 2005b; (4) Krimm et al. 2005; (5) Berger et al. 2005; (6) Cummings et al. 2005; (7) Soderberg et al. 2006; (8) de Ugarte Postigo et al. 2006; (9) Berger 2010a; (10) Markwardt et al. 2006; (11) Roming et al. 2006; (12) Urata et al. 2006; (13) Schady et al. 2006; (14) Berger et al. 2007; (15) D’Avanzo et al. 2009; (16) Marshall et al. 2006; (17) Stratta et al. 2007; (18) Markwardt et al. 2007; (19) Cenko et al. 2008; (20) Gotz et al. 2007; (21) Piranomonte et al. 2008; (22) Kodaka et al. 2007; (23) Racusin et al. 2007; (24) Ziaeepour et al. 2007; (25) Berger et al. 2009; (26) Marshall et al. 2007; (27) Sato et al. 2007b; (28) D’Avanzo et al. 2007; (29) Sakamoto et al. 2007; (30) Mao et al. 2008; (31) Perley et al. 2009; (32) Pagani et al. 2008; (33) Rowlinson et al. 2010; (34) Krimm et al. 2008; (35) Nicuesa Guelbenzu et al. 2012; (36) Krimm et al. 2009; (37) Antonelli et al. 2009; (38) Levesque et al. 2010; (39) Hoversten et al. 2009; (40) McBreen et al. 2010; (41) Barthelmy et al. 2009; (42) Oates et al. 2009; (43) Levan et al. 2009; (44) de Pasquale et al. 2010; (45) Fong et al. 2011; (46) Barthelmy et al. 2011; (47) Sakamoto et al. 2011; (48) Fong et al. 2012; (49) Sakamoto et al. 2013; (50) Margutti et al. 2012; (51) Gehrels et al. 2005; (52) Bloom et al. 2006; (53) Sato et al. 2005; (54) Berger 2005; (55) Foley et al. 2005; (56) Berger 2006; (57) Prochaska et al. 2006; (58) La Parola et al. 2006; (59) Sato et al. 2006a; (60) Bloom et al. 2007; (61) Sato et al. 2006b; (62) Berger 2009; (63) Cannizzo et al. 2006; (64) Ziaeepour et al. 2006; (65) Sato et al. 2007a; (66) Leibler \& Berger 2010; (67) Uehara et al. 2008; (68) Ukwatta et al. 2008; (69) Krimm et al. 2010b; (70) Perley et al. 2012; (71) Holland et al. 2010b; (72) Krimm et al. 2010a. 
Table 4

Short GRB Environment Distributions

\begin{tabular}{|c|c|c|c|c|c|c|c|c|}
\hline Sample & Late-type & Early-type & Inconclusive & Host-less & Total & $\mathrm{L}: \mathrm{E}$ ratio $^{\mathrm{a}}$ & $P_{\text {binom }}(\geqslant \mathrm{L}: \mathrm{E})^{\mathrm{b}}$ & Reject 1:1 Distribution? ${ }^{\mathrm{c}}$ \\
\hline Subarcsec. & $11(44 \%)$ & $2(8 \%)$ & $6(24 \%)$ & $6(24 \%)$ & 25 & $5.5: 1$ & 0.01 & Yes \\
\hline Subarcsec., Host-less assigned & $12(48 \%)$ & $4(16 \%)$ & $9(36 \%)$ & & 25 & $3: 1$ & 0.04 & Yes, marginal \\
\hline Subarcsec. + XRT & $17(47 \%)$ & $6(17 \%)$ & $7(19 \%)$ & $6(17 \%)$ & 36 & $2.8: 1$ & 0.02 & Yes \\
\hline Subarcsec. + XRT, Host-less assigned & $18(50 \%)$ & $8(22 \%)$ & $10(28 \%)$ & & 36 & $2.3: 1$ & 0.04 & Yes, marginal \\
\hline Subarcsec. + XRT, All Inc. are Early-type & $18(50 \%)$ & $18(50 \%)$ & & & 36 & $1: 1$ & 0.5 & No \\
\hline Subarcsec. + XRT, EE excluded & $12(43 \%)$ & $7(25 \%)$ & $9(32 \%)$ & & 28 & $1.7: 1$ & 0.19 & No \\
\hline Subarcsec. + XRT, $P_{\mathrm{NC}}>0.9$ & $8(58 \%)$ & $3(21 \%)$ & $3(21 \%)$ & & 14 & $2.7: 1$ & 0.11 & No \\
\hline
\end{tabular}

Notes.

${ }^{\text {a }}$ Late-to-early-type ratio.

${ }^{\mathrm{b}} p$ value for finding greater than or equal to the observed L:E ratio from a 1:1 binomial distribution.

c Assumes a significance level of 0.05 .

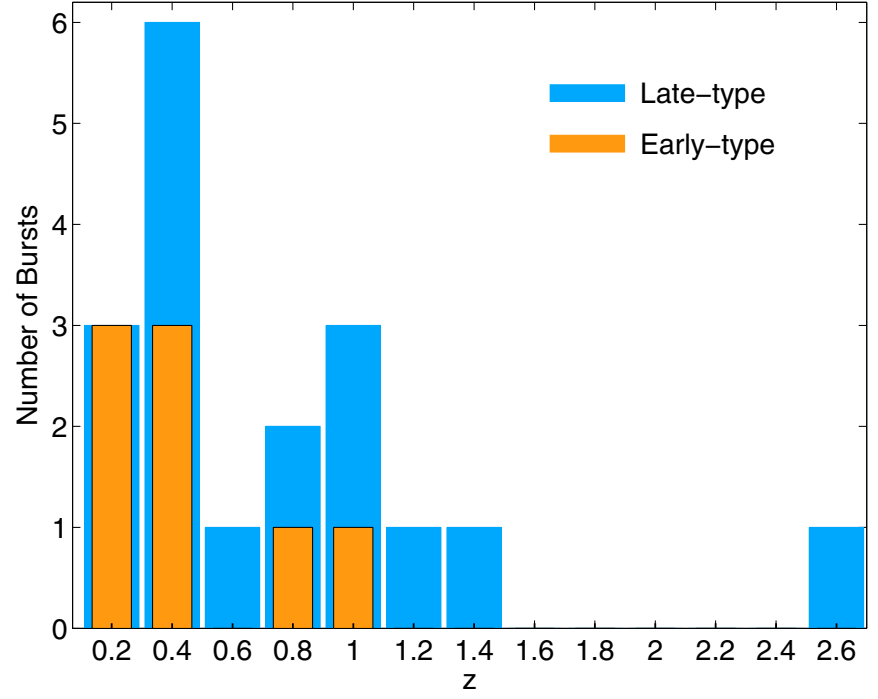

Figure 13. Redshift distribution of 26 short GRBs with host associations and redshifts, classified by type of the host galaxy, either late-type (blue) or earlytype (orange). Redshifts and classifications are based on spectroscopy, with the exception of two hosts, GRBs 070729 and 111117A, which are based on wellsampled broadband photometry (Table 3; Leibler \& Berger 2010; Margutti et al. 2012).

(A color version of this figure is available in the online journal.)

GRBs that have relatively small offsets of $\approx 1 \mathrm{kpc}$ (Bloom et al. 2002; Fruchter et al. 2006). From afterglow observations, the inferred densities for these three events may span a wide range, $n_{0} \sim 10^{-4}-1 \mathrm{~cm}^{-3}$, while long GRBs have values of $n_{0} \gtrsim 0.1 \mathrm{~cm}^{-3}$ (Soderberg et al. 2006).

The host galaxies of GRBs 100625A and 101219A have stellar populations that span the observed distribution of short GRB hosts. With $\tau \approx 25-100 \mathrm{Myr}$ and $\log \left(M_{*} / M_{\odot}\right) \approx 9.1$, GRB 101219A is at the low end of both the short GRB age and mass distributions (Leibler \& Berger 2010). This host also has one of the most vigorous SFRs reported for a short GRB host to date (Berger 2009; Perley et al. 2012; Berger et al. 2013), and an appreciable extinction of $A_{V}^{\text {host }} \gtrsim 2$ mag. These characteristics match more closely with the median parameters observed for long GRB host galaxies (Christensen et al. 2004; Wainwright et al. 2007; Leibler \& Berger 2010). However, an independent study based on the $\gamma$-ray properties reports a probability that GRB 101219A is not a collapsar of 94\% (Bromberg et al. 2013). Compared to other early-type hosts, GRB 100625A has a similar age (0.6-0.8 Gyr) and SFR limit (Bloom et al. 2006; Berger 2009; Leibler \& Berger 2010; Fong et al. 2011), but its stellar mass, $\log \left(M_{*} / M_{\odot}\right) \approx 9.7$, is the lowest by an order of magnitude (Leibler \& Berger 2010).

\section{HOST GALAXY DEMOGRAPHICS}

To broadly determine and utilize the short GRB host population, we expand upon the observations presented here and investigate the demographics of the bulk of the Swift short GRB sample, quantifying the fractions of events that explode in different types of environments. We divide the population into four host galaxy categories: late-type, early-type, inconclusive (coincident hosts that are too faint to classify as late- or early-type), and "host-less" (lack of coincident hosts to $\gtrsim 26 \mathrm{mag}$ ). All lateand early-type designations are based on spectroscopic classification, with the exception of two hosts, GRBs 070729 and 111117A, which are based on well-sampled broadband photometry (Table 3; Leibler \& Berger 2010; Margutti et al. 2012).

We then use our classifications to examine the relative rates of short GRBs detected in early- and late-type galaxies. In the absence of observational selection effects, if the overall short GRB rate tracks stellar mass alone, the relative detection rates in early- and late-type galaxies should match the distribution of stellar mass, which is roughly equal at $z \sim 0$ (Kochanek et al. 2001; Bell et al. 2003; Driver et al. 2007) and shows little evolution to $z \sim 1$ (Ilbert et al. 2010). On the other hand, if the short GRB rate depends on a combination of stellar mass and star formation, as in the case of Type Ia SNe (Sullivan et al. 2006), we expect a distribution skewed toward star-forming galaxies, with a late-to-early-type ratio of $>1: 1$.

\subsection{Environment Fractions}

We first analyze the subset of bursts with subarcsecond localization because they have the most unambiguous associations. Of the 68 short GRBs detected with Swift ${ }^{12}$ as of 2012 May, there are 25 such events (Table 3), 2 of which have been localized with Chandra (GRB 111020A: Fong et al. 2012; GRB 111117A: Margutti et al. 2012; Sakamoto et al. 2013), an alternative route to subarcsecond positions in the absence of an optical afterglow. This population is divided as follows: $11(44 \%)$ originate in latetype galaxies, $2(8 \%)$ are in early-type galaxies, $6(24 \%)$ have hosts of inconclusive type, and $6(24 \%)$ are host-less (Berger 2010a and this work; Figure 14 and Table 4). From probability of chance coincidence arguments, we can assign the six host-less GRBs to a most probable host galaxy. Berger (2010a)

\footnotetext{
12 We note that two of the bursts in our sample, GRBs 050709 and 060121 , were first discovered by the High Energy Transient Explorer 2 (HETE-2) satellite.
} 

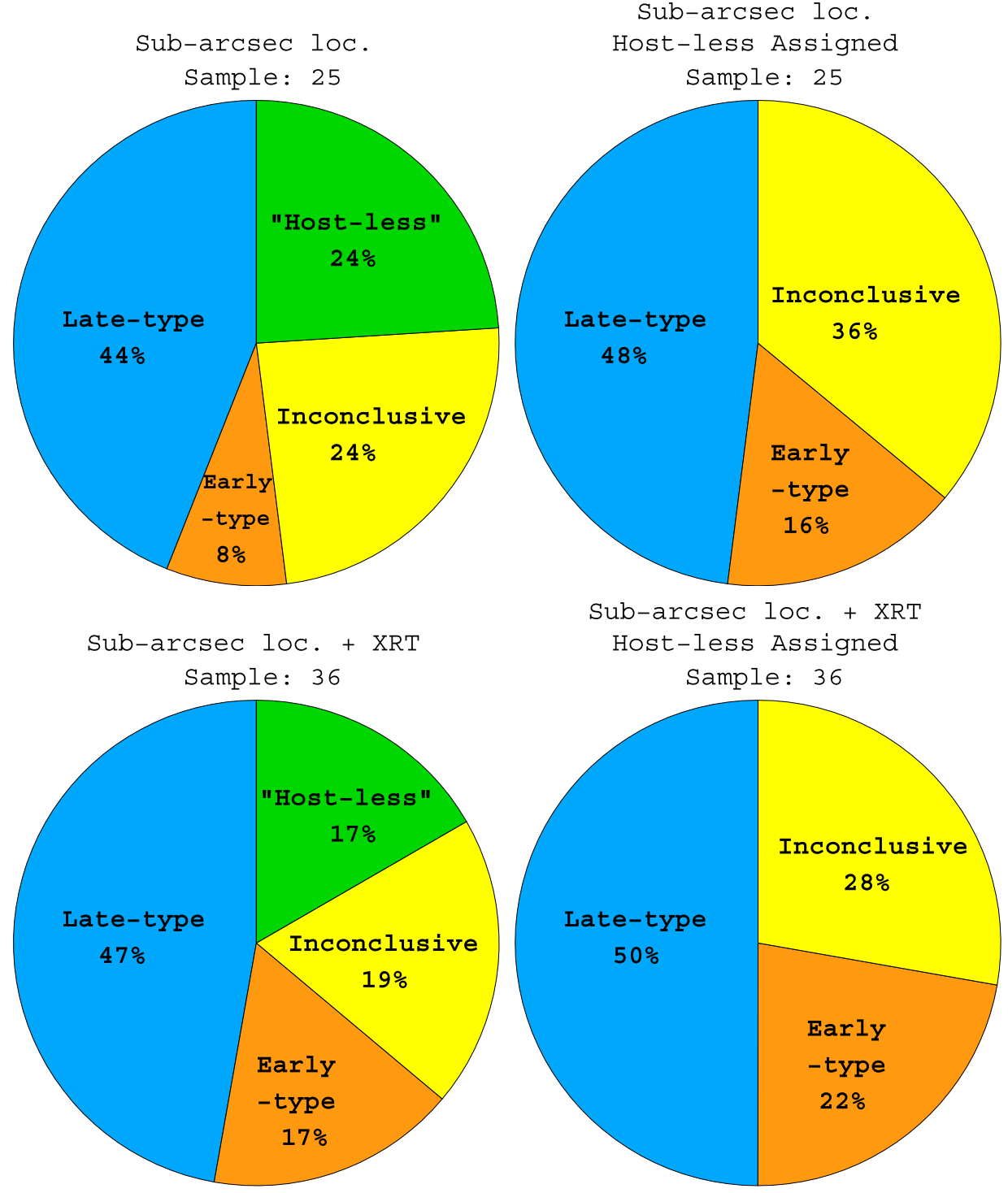

Sub-arcsec loc. + XRT, no EE Host-less Assigned Sample: 28

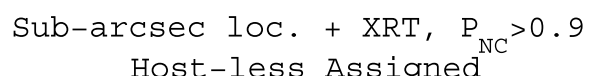
Host-less Assigned
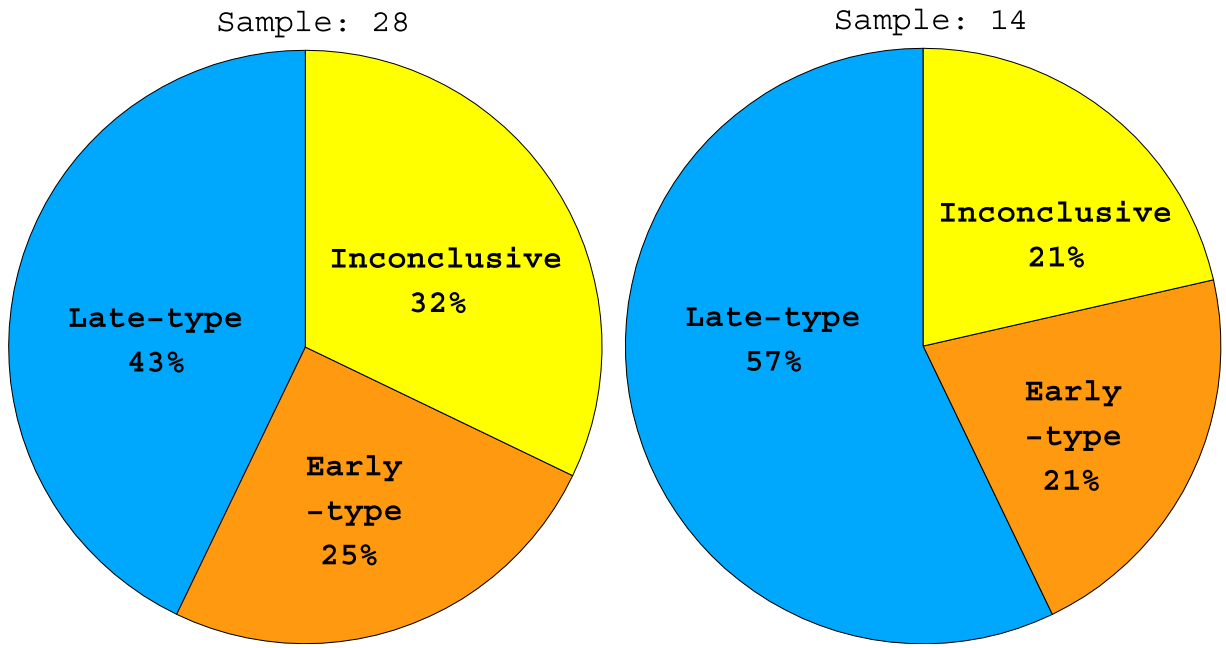

Figure 14. Distribution of short GRB environments, according to Table 3. The fractions of late-type (blue), early-type (orange), host-less (green) and inconclusive (yellow) environments are shown. Top: the distribution of 25 short GRBs with subarcsecond localization is divided into all four categories (left), and the 6 host-less bursts are each assigned to their most probable host galaxy (right; Berger 2010a and this work). Middle: our full sample, including 11 short GRBs with XRT localizations and probable hosts, is divided into all four categories (left), and with the 6 host-less bursts assigned (right). Bottom: distribution of our sample for which there is no evidence for extended emission (left) and for which $P_{\mathrm{NC}}>0.9$ (right; Bromberg et al. 2013).

(A color version of this figure is available in the online journal.) 
investigated five events, finding two that likely originated in early-type hosts (GRBs 070809 and 090515), one with a latetype host (GRB 061201), and two with hosts of inconclusive type (GRBs 080503 and 090305). We have shown that the remaining host-less burst, GRB 110112A, lacks an obvious host galaxy (Section 4.3), and we classify it as inconclusive.

Accounting for these host-less assignments in the distribution of galaxy types, we do not find a substantial change in the relative fractions (Figure 14). Considering the 16 bursts with definitive host types, the late-to-early-type ratio is $3: 1$, which deviates from the expected 1:1 distribution if the short GRB rate depends only on stellar mass. Using binomial statistics, we test the null hypothesis of a distribution that is intrinsically $1: 1$ and find that the observed ratio has a $p$-value of only 0.04 , indicating that the null hypothesis is disfavored (Table 4).

Because the optical afterglow brightness depends on the circumburst density, $n_{0}$ (Granot \& Sari 2002), the requirement of an optical afterglow for precise positions (with the exception of the two bursts localized by Chandra) may affect the relative rates of short GRB detection in early- and late-type hosts if there is a correlation between average density and galaxy type. To assess this potential effect, we broaden our analysis to include bursts with a single probable host galaxy $\left(P_{\mathrm{cc}}(<\delta R) \lesssim 0.05\right)$ within or on the outskirts of XRT error circles. This sample comprises 11 additional events ${ }^{13}$ with localizations of $1^{\prime \prime} .5-5^{\prime \prime} .5$ in radius (90\% containment, Table 3), bringing the total sample size to 36 bursts. Since we require subarcsecond localization for a burst to be classified as host-less, the relative fraction of these events is artificially diluted by the addition of bursts with XRT positions (Figure 14).

Assigning the host-less bursts to their most probable host galaxies, we recover a similar distribution to the subarcsecond localized sample: $\approx 50 \%$ late-type, $\approx 20 \%$ early-type, and $\approx 30 \%$ inconclusive (Figure 14 and Table 4). Based on the 26 bursts with early- and late-type designations, this gives a lateto-early-type ratio of 2.3:1 and a low $p$-value of 0.04 for the null hypothesis that this distribution is drawn from an intrinsically 1:1 distribution. To directly compare this $2.3: 1$ ratio to the $3: 1$ observed ratio for subarcsecond localized bursts, we compute the probability of obtaining a ratio $\leqslant 2.3: 1$ from a population with a true ratio of 3:1 using Monte Carlo simulations for the binomial distribution. In $10^{5}$ trials, we calculate a high probability of 0.82 , suggesting that there is no bias to the environment fractions when analyzing only subarcsecond localized bursts.

Next, we address the remaining population of 32 Swift short GRBs excluded from the discussion thus far. The majority, $80 \%$, are affected by observing constraints that are dependent on factors completely decoupled from any intrinsic properties of the bursts: 15 had Swift re-pointing constraints (Sun or Moon) and thus have only $\gamma$-ray positions, 7 have XRT positions that are highly contaminated (in the direction of the Galactic plane or near a saturated star, e.g., GRB 100702A, see Appendices A and B), and 4 have XRT afterglows but so far lack adequate optical/NIR follow-up to determine the presence of a host galaxy; thus, we cannot currently distinguish between a faint coincident host and a host-less origin for these 4 bursts. The remaining $20 \%$ (six events) have no XRT localization despite rapid $S$ wift re-pointing ( $\delta t \lesssim 2$ minutes), but have a low median fluence of $f_{\gamma} \approx 2 \times 10^{-8} \mathrm{erg} \mathrm{cm}^{-2}$ compared to the rest of the population with $\left\langle f_{\gamma, \mathrm{SGRB}}\right\rangle \approx 2 \times 10^{-7} \mathrm{erg} \mathrm{cm}^{-2}(15-150 \mathrm{keV}$; Figure 15). Therefore, the lack of detectable emission with

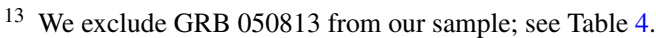

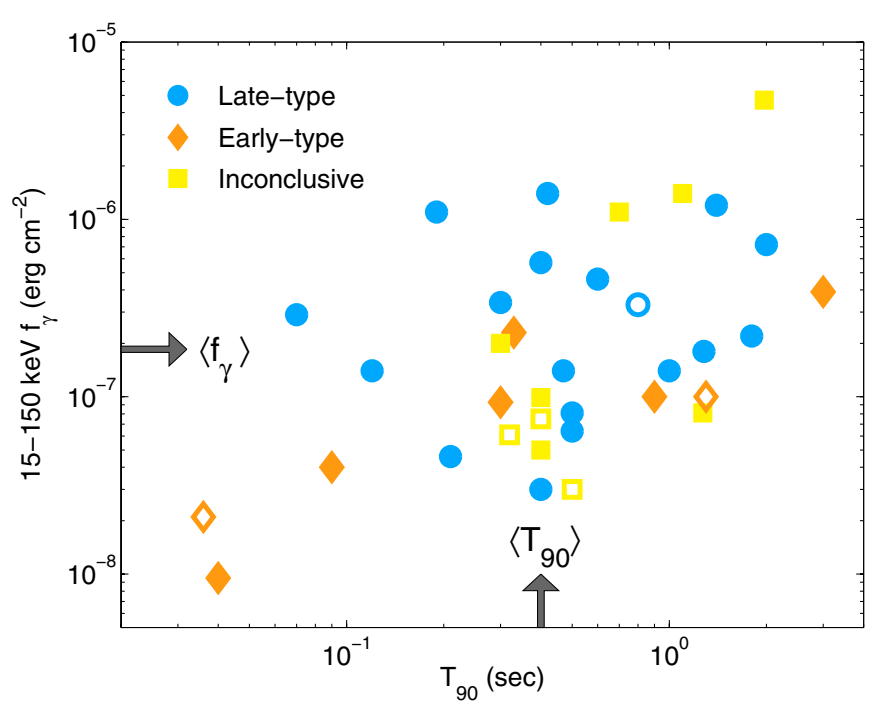

Figure 15. Fluence, $f_{\gamma}(15-150 \mathrm{keV})$, vs. duration, $T_{90}$, for the subarcsecond localized + XRT sample of 36 Swift short GRBs. Bursts are classified by morphological type (Table 3) as late-type (blue), early-type (orange), and inconclusive (yellow). Open symbols denote host-less assignments. The median

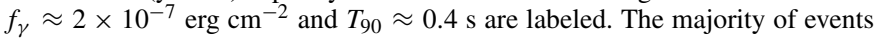

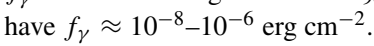

(A color version of this figure is available in the online journal.)

XRT may be related to an intrinsically lower energy scale. In summary, we do not expect the exclusion of these 32 bursts to have a substantial effect on the relative morphological fractions.

The low observed early-type fraction is likely attributed to one of two possibilities: (1) it is more challenging to identify earlytype galaxies at higher redshifts, and thus a disproportionate fraction of the bursts designated as inconclusive are in fact earlytype; or (2) short GRBs preferentially occur in late-type galaxies due to the intrinsic properties of their progenitors.

We explore the former option by investigating the inconclusive population in more detail. SEDs of early-type galaxies generally lack strong emission lines, and the most prominent features, the $4000 \AA$ break and the Ca II H\&K absorption lines, are redshifted out of the range of most optical spectrographs for $z \gtrsim 1.5$, making spectroscopic identifications particularly difficult at these redshifts. However, more effective studies selecting for distant early-type field galaxies by their photometric optical/ NIR colors detect a nearly constant number of early-types between $z \approx 1$ and 1.5 (Stanford et al. 2004), with a typical AB color of 1-4 mag, depending on the choice of optical/NIR filters (Stanford et al. 2004; Tamura \& Ohta 2004). Of the 10 inconclusive host galaxies, 4 have optical/NIR color information but yield only poor constraints of $\lesssim 3-5$ mag due to NIR non-detections and faint optical magnitudes, and 5 lack reported NIR observations. The only inconclusive host galaxy with multiband detections, GRB 060121, has $R-H \approx 2.4$ mag; however, the optical afterglow and objects in the vicinity are comparably red, suggesting a $z>2$ origin as an explanation for the red host color (Levan et al. 2006a). $K$-band imaging to depths of $\gtrsim 23 \mathrm{AB}$ mag might enable progress in deducing what fraction of the inconclusive population is more likely early-type. To set an extreme upper bound on the true early-type fraction, if we assume that all inconclusive hosts are early-types, the projected early-type fraction is $\sim 50 \%$ (Table 4 ).

We now turn to the second option, that short GRBs preferentially originate from late-type galaxies. The predicted demographics of NS-NS/NS-BH merger populations show a 
preference toward late-type galaxies of $\gtrsim 70 \%$ (O'Shaughnessy et al. 2008), so we can use the observed short GRB population to assess the implications for the progenitors. We expect to find roughly equal early- and late-type fractions if stellar mass is the sole parameter determining the short GRB rate. However, we only observe this for $z<0.4$ (six events; Figure 13). For $z>0.4$, the late-type fraction is consistently higher, with a late-to-early-type ratio of $\gtrsim 2: 1$. These results, along with the previous finding that the short GRB rate per unit stellar mass is 2-5 times higher in late-type hosts (Leibler \& Berger 2010), suggest that the short GRB rate is dependent upon a combination of stellar mass and star formation. In the context of NS-NS/NS-BH mergers, if the delay times of the systems that give rise to short GRBs are very long ( $\gtrsim$ few Gyr), we would expect a dominant population of early-type hosts at $z \sim 0$. Instead, the current demographics show a preference for late-type galaxies.

Along with the inferred stellar population ages from SED modeling (Leibler \& Berger 2010), this suggests moderate delay times of $\lesssim$ few Gyr. For a delay time distribution of the form $P(\tau) \propto \widetilde{\tau^{n}}$, this translates to $n \lesssim-1$, in contrast to previous short GRB results that claimed substantially longer average delay times of $\sim 4-8$ Gyr for lognormal lifetime distributions based on smaller numbers of events (Nakar et al. 2006; Zheng \& Ramirez-Ruiz 2007; Gal-Yam et al. 2008). These results are in good agreement with predictions for populations of NS-NS/NS-BH mergers from population synthesis (Dominik et al. 2012). We also note the similarity to the delay time distribution of Type Ia SNe, which have $n \approx-1.1$ (Maoz et al. 2010, 2012).

In summary, we find that unless all inconclusive hosts are early-type, the short GRB host distribution is skewed toward late-type galaxies, with the most likely ranges for the early- and late-type fractions of $\approx 20 \%-40 \%$ and $\approx 60 \%-80 \%$, respectively, for the entire short GRB population. Furthermore, for most cuts on the sample we find that the null hypothesis of a 1:1 distribution can be mildly or strongly rejected.

\subsection{Comparison with $\gamma$-Ray Properties}

We next investigate whether there is contamination in our sample from collapsars by analyzing trends between morphological type and $\gamma$-ray properties. We find that bursts in earlyand late-type galaxies span the entire distribution of observed $T_{90}$ for short GRBs, with a median value of $0.4 \mathrm{~s}$ (Figure 15). Using a Kolmogorov-Smirnov (K-S) test, we find that the two populations are consistent with being drawn from the same underlying distribution ( $p=0.43$ ). The claim becomes stronger when we compare the combined early-type and inconclusive distribution with the late-type distribution $(p=0.94)$. On the other hand, the corresponding K-S tests for the fluence distributions (Figure 15) yield marginal $p$-values of 0.05 , suggesting that bursts associated with early- and late-types may not be drawn from the same underlying distribution in $f_{\gamma}$.

A recent study by Bromberg et al. (2013) used the $\gamma$-ray properties ( $T_{90}$ and spectral hardness) to derive a probability that each event is not a collapsar $\left(P_{\mathrm{NC}}\right)$, excluding eight bursts that have reported evidence for extended emission. Of the 29 bursts that overlap in our samples, 14 have a high probability of not arising from a collapsar $\left(P_{\mathrm{NC}}>0.9\right)$. If these probabilities are robust, and there is contamination from collapsars in our full sample, we would expect the galaxy type fractions for the population with $P_{\mathrm{NC}}>0.9$ to differ from the overall sample. In particular, by including only high-probability non-collapsar events, we would presumably be excluding mostly late-type galaxies since all long GRBs/collapsars are found in starforming galaxies. Therefore, one would naively expect the lateto-early-type ratio to decrease with respect to the full sample. However, we find that the late-to-early-type ratio for this sample is $2.7: 1$ (Table 4; Figure 14), which is higher than the 2.3:1 ratio inferred for the sample of 36 short GRBs.

However, $P_{\mathrm{NC}}$ values are not reported for bursts with extended emission. Thus, for a more direct comparison, we evaluate the subset of 28 short GRBs without extended emission (Figure 14) and calculate a late-to-early-type ratio of 1.7:1 (Table 4). Interestingly, all bursts with extended emission originate in late-type (or inconclusive) galaxies, with the exception of GRB 050724A. Since the ratio for the $P_{\mathrm{NC}}>0.9$ population is more skewed toward late-type galaxies with $2.7: 1$, the probability of obtaining a $\geqslant 2.7: 1$ ratio in 14 events from an intrinsically $1: 7: 1$ distribution is moderate, 0.37 . This not only demonstrates no noticeable contamination to the short GRB host type distribution when including bursts with reportedly high probabilities of being collapsars, but also calls into question the reliability or importance of these probabilities in assessing the true population of short GRBs.

\section{CONCLUSIONS}

We present broadband observations of three short GRBs: GRB 100625A associated with an early-type galaxy at $z=$ 0.452 , GRB 101219A associated with an active star-forming galaxy at $z=0.718$, and GRB 110112A, which has a subarcsecond localization from an optical afterglow but no coincident host galaxy to deep optical limits, and no convincing putative host within $5^{\circ}$ of the burst location. These observations showcase the diversity of short GRB environments and give direct clues to the nature of the short GRB progenitor: the moderate physical offsets and low inferred densities can be interpreted as evidence for a compact binary progenitor.

We also undertake the first comprehensive study of host demographics for the full Swift short GRB population, classifying bursts by their host galaxy type. We emphasize several key conclusions:

1. The sample of subarcsecond localized bursts has a host galaxy distribution of $\approx 50 \%$ late-type, $\approx 20 \%$ early-type, and $\approx 30 \%$ inconclusive type after assigning host-less bursts. The inclusion of bursts with Swift/XRT positions and convincing host associations $\left(P_{\mathrm{cc}}(<\delta R) \lesssim 0.05\right)$ does not affect the relative fractions.

2. The observed late-to-early-type ratio is $\gtrsim 2: 1$, and most cuts to the sample demonstrate that an intrinsically 1:1 distribution is improbable. The only way to obtain equal fractions with the observed events is by assuming that all inconclusive hosts are early-type galaxies at $z \gtrsim 1$.

3. The most likely ranges for the early- and late-type fractions are $\approx 20 \%-40 \%$ and $\approx 60 \%-80 \%$. The preference toward late-type galaxies suggests that both stellar mass and star formation play roles in determining the short GRB rate. Furthermore, in the context of the NS-NS/NS-BH mergers, the observed short GRB population is not dominated by systems with very long delay times, but instead with typical delay times of $\lesssim$ few Gyr.

4. There is no clear trend between $T_{90}$ and host galaxy type, while there may be a relationship between $f_{\gamma}$ and host type. When excluding the population of bursts reported to 
be likely collapsars ( $>90 \%$ probability), the late-type fraction increases relative to the overall short GRB sample, suggesting that these probabilities are not reliable in assessing the true population.

Looking forward, our study has demonstrated that detailed observations of short GRB afterglows and environments hold the key to understanding the underlying population of progenitors. In particular, we emphasize the importance of deep NIR observations to determine the early-type fraction within the inconclusive population of hosts, and Hubble Space Telescope observations of short GRBs that lack coincident host galaxies to ground-based optical limits ( $\approx 26 \mathrm{mag})$. A concerted analysis of broadband short GRB afterglows would complement this study by providing constraints on the basic properties of the bursts (i.e., energy scale, circumburst density) and help to determine whether there are any correlations between these basic properties and galactic environment. Finally, these updated short GRB demographics now enable a more detailed comparison to published theoretical predictions for the relative fractions of early- and late-type galaxies that host NS-NS/NS-BH mergers (O'Shaughnessy et al. 2008) and their delay time distributions (Dominik et al. 2012).

We thank F. di Mille for observing on behalf of the Berger GRB group at Harvard. The Berger GRB group is supported by the National Science Foundation under grant AST-1107973, and by NASA/Swift AO6 grant NNX10AI24G and A07 grant NNX12AD69G. Partial support was also provided by the National Aeronautics and Space Administration through Chandra Award Number GO1-12072X issued by the Chandra X-Ray Observatory Center, which is operated by the Smithsonian Astrophysical Observatory for and on behalf of the National Aeronautics Space Administration under contract NAS8-03060. This paper includes data gathered with the $6.5 \mathrm{~m}$ Magellan Telescopes located at Las Campanas Observatory, Chile. This work is based in part on observations obtained at the Gemini Observatory, which is operated by the Association of Universities for Research in Astronomy, Inc., under a cooperative agreement with the NSF on behalf of the Gemini partnership: the National Science Foundation (United States), the Science and Technology Facilities Council (United Kingdom), the National Research Council (Canada), CONICYT (Chile), the Australian Research Council (Australia), Ministério da Ciência, Tecnologia e Inovação (Brazil), and Ministerio de Ciencia, Tecnología e Innovación Productiva (Argentina). This work made use of data supplied by the UK Swift Science Data Centre at the University of Leicester. S.B.C. acknowledges generous support from Gary \& Cynthia Bengier, the Richard \& Rhoda Goldman Fund, the Christopher R. Redlich Fund, the TABASGO Foundation, and NSF grants AST-0908886 and AST-1211916.

\section{APPENDIX A \\ GRB 100628A}

GRB 100628A was detected by Swift/BAT and the AntiCoincidence System on International Gamma-Ray Astrophysics Laboratory on 2010 June 28.345 UT with $T_{90}=0.036 \pm 0.009 \mathrm{~s}$ $(15-350 \mathrm{keV}), f_{\gamma}=(2.5 \pm 0.5) \times 10^{-8} \mathrm{erg} \mathrm{cm}^{-2}(15-150 \mathrm{keV})$, and peak energy $E_{\text {peak }}=74.1 \pm 11.4 \mathrm{keV}$. The groundcalculated position is R.A. $=15^{\mathrm{h}} 03^{\mathrm{m}} 46^{\mathrm{s}} \cdot 2$, decl. $=-31^{\circ} 39^{\prime} 10^{\prime} \cdot 2$ with an uncertainty of 2!.1 (Immler et al. 2010).

\section{A.1. X-Ray Observations}

XRT began observing the field at $\delta t=86 \mathrm{~s}$ and detected an $\mathrm{X}$-ray source in coincidence with the core of a bright galaxy. The lack of fading of this source confirmed by Chandra/ ACIS-S observations at $\delta t=4.4$ days suggests an AGN origin (Immler et al. 2010; Berger 2010b). Furthermore, we use binomial statistics and a 10-pixel region centered on the source to calculate the probability of a chance fluctuation, finding a high probability of $15 \%$. Thus, this source is ruled out as the afterglow of GRB 100628A. A second candidate afterglow was reported based on seven counts over $3.8 \mathrm{ks}$ in the time interval $\delta t=92-7200 \mathrm{~s}$, which translates to a count rate of $0.0017_{-0.0006}^{+0.0008}$ counts s ${ }^{-1}(0.3-10 \mathrm{keV}$; Immler et al. 2010). UVOT, which commenced observations at $\delta t=90 \mathrm{~s}$, did not detect a coincident source to $\gtrsim 20.2$ mag (white filter; Immler et al. 2010).

We re-analyze the same time interval of XRT data and use the ximage routine in the HEASOFT package to measure the significance of the source. In a blind search, we find the source has a significance of $2.3 \sigma$. Late-time XRT and Chandra observations confirm that the source has faded by a factor of $\sim 15$ from the claimed initial X-ray flux (Berger 2010b). However, we do not include this burst in our sample of short GRBs with XRT positions due to the low significance of the initial source. We caution against classifying this burst as XRT-localized in future short GRB samples.

\section{APPENDIX B}

\section{GRB 100702A}

Swift/BAT detected GRB 100702A on 2010 July 02.044 UT with $T_{90}=0.16 \pm 0.03 \mathrm{~s}(15-350 \mathrm{keV})$ and $f_{\gamma}=(1.2 \pm$ $0.1) \times 10^{-7}$ erg cm$~^{-2}(15-150 \mathrm{keV})$ at a ground-calculated position of R.A. $=16^{\mathrm{h}} 22^{\mathrm{m}} 46^{\mathrm{s}} .4$ and decl. $=-56^{\circ} 32^{\prime} 57^{\prime \prime} .4$ with an uncertainty of 1.4 in radius (Siegel et al. 2010).

\section{B.1. X-Ray Observations}

XRT started observing the field at $\delta t=94 \mathrm{~s}$ and identified a fading X-ray counterpart with a final UVOT-enhanced positional accuracy of 2".4 (Table 1; Goad et al. 2007; Evans et al. 2009). UVOT commenced observations at $\delta t=101 \mathrm{~s}$ and no source was identified in the white filter to a limit of $\gtrsim 18 \mathrm{mag}$ (Siegel et al. 2010). The XRT light curve is best fit with a broken power law with decay indices of $\alpha_{X, 1}=-0.86_{-0.24}^{+0.17}$ and $\alpha_{X, 2}=-5.04_{-0.37}^{+0.34}$ and a break time at $\delta t=202 \mathrm{~s}$ (Evans et al. 2009).

We extract a spectrum from the XRT data (method described in Section 2.1.1) and utilize the full PC data set, where there is no evidence for spectral evolution. Our best-fit model is characterized by $\Gamma=2.7 \pm 0.3$ and $(4.4 \pm 2.0) \times 10^{21} \mathrm{~cm}^{-2}$ in excess of the substantial Galactic value, $N_{\mathrm{H}, \mathrm{MW}}=2.8 \times$ $10^{21} \mathrm{~cm}^{-2}$ (Kalberla et al. 2005). We note that the burst is in the direction of the Galactic center $(b=-4.8)$ and therefore the uncertainties on $N_{\mathrm{H}, \mathrm{MW}}$ are likely larger than the typical $10 \%$. Our results are consistent with the automatic fits by Evans et al. (2009).

\section{B.2. Optical/NIR Observations and Afterglow Limits}

We obtained $J$-band observations of the field of GRB 100702A with PANIC at $\delta t=1.3 \mathrm{hr}$ (Figure 16). We detect four sources within or near the outskirts of the XRT error 


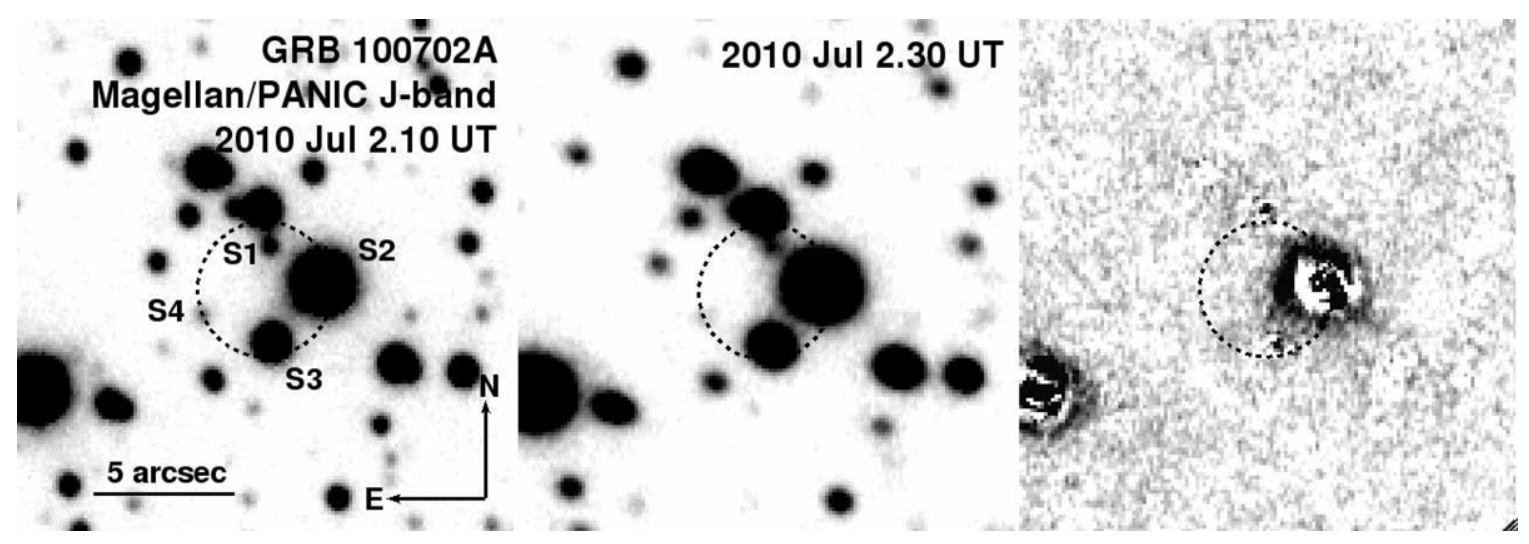

Figure 16. Magellan/PANIC $J$-band observations of the host galaxy of GRB 100702A. The XRT error circle has a radius of 2". 4 (90\% containment; black). Left: $\delta t=1.3 \mathrm{hr}$. Center: $\delta t=6.1 \mathrm{hr}$. Right: digital image subtraction of the two epochs reveals no afterglow to a $3 \sigma$ limit of $J \gtrsim 23.3 \mathrm{mag}$.

circle (S1-S4 in Figure 16). S2 and S3 have stellar point-spread functions (PSFs), while S1 and S4 have non-stellar PSFs. Previously reported $J$-band observations also confirm that $\mathrm{S} 2$ and S3 are stars (Nicuesa Guelbenzu et al. 2012), while S1 and S4 have not been reported in the literature. ${ }^{14}$ To assess any fading within the XRT position, we obtained a second set of $J$-band observations at $\delta t=6.1 \mathrm{hr}$. Digital image subtraction reveals no residuals to a $3 \sigma$ limit of $J \gtrsim 23.3 \mathrm{mag}$ (Table 2). We caution that this limit only applies to $2 / 3$ of the error circle due to contamination from the saturated star, S2 (Figure 16). In addition, we obtained $i$-band observations with IMACS at $\delta t=247.3$ days and we do not detect any additional sources in or around the XRT error circle (Table 2).

\section{B.3. Probabilities of Chance Coincidence}

We calculate $P_{\mathrm{cc}}(<\delta R)$ for $\mathrm{S} 1$ and $\mathrm{S} 4$ to assess either source as a putative host galaxy for GRB 100702A. Source S1 is fully inside the XRT error circle while S4 lies on the outskirts of the XRT error circle. We perform PSF photometry for both sources (Table 2) and calculate their probabilities of chance coincidence: $P_{\mathrm{cc}}(<\delta R) \approx 0.02$ for $\mathrm{S} 1$ and $P(<\delta R) \approx 0.04$ for S2 using the $3 \sigma$ XRT position radius of 4".5. This analysis suggests that either source is a likely host for GRB 100702A, and we cannot currently distinguish which is more likely. We also note that the significant contamination makes it difficult to exclude the possibility that there is a brighter galaxy within the XRT error circle. Therefore, we do not include GRB 100702A in our sample of bursts with XRT localization, and we consider this field to have observing constraints that prevent more in-depth analysis.

\section{REFERENCES}

Alard, C. 2000, A\&AS, 144, 363

Anderson, J. P., Habergham, S. M., James, P. A., \& Hamuy, M. 2012, MNRAS, 424, 1372

Antonelli, L. A., D’ Avanzo, P., Perna, R., et al. 2009, A\&A, 507, L45

Barthelmy, S. D., Baumgartner, W. H., Beardmore, A. P., et al. 2009, GCN, 9364, 1

Barthelmy, S. D., Sakamoto, T., \& Stamatikos, M. 2011, GCN, 11557, 1

Belczynski, K., Perna, R., Bulik, T., et al. 2006, ApJ, 648, 1110

Bell, E. F., McIntosh, D. H., Katz, N., \& Weinberg, M. D. 2003, ApJS, 149, 289

Berger, E. 2005, GCN, 3801, 1

Berger, E. 2006, in AIP Conf. Ser. 836, Gamma-Ray Bursts in the Swift Era, ed. S. S. Holt, N. Gehrels, \& J. A. Nousek (Melville, NY: AIP), 33

\footnotetext{
14 Our PANIC observations show that source "C" in Nicuesa Guelbenzu et al. (2012) is actually three blended sources, including S1. The remaining two sources are outside of the XRT error circle.
}

Berger, E. 2009, ApJ, 690, 231

Berger, E. 2010a, ApJ, 722, 1946

Berger, E. 2010b, GCN, 10942, 1

Berger, E., Cenko, S. B., Fox, D. B., \& Cucchiara, A. 2009, ApJ, 704, 877

Berger, E., Fox, D. B., Price, P. A., et al. 2007, ApJ, 664, 1000

Berger, E., Price, P. A., Cenko, S. B., et al. 2005, Natur, 438, 988

Berger, E., Zauderer, B. A., Levan, A. J., et al. 2013, ApJ, 765, 121

Bhat, P. N. 2010, GCN, 10912, 1

Bloom, J. S., Kulkarni, S. R., \& Djorgovski, S. G. 2002, AJ, 123, 1111

Bloom, J. S., Perley, D. A., Chen, H.-W., et al. 2007, ApJ, 654, 878

Bloom, J. S., Prochaska, J. X., Pooley, D., et al. 2006, ApJ, 638, 354

Breeveld, A. A., \& Stamatikos, M. 2011, GCN, 11560, 1

Bromberg, O., Nakar, E., Piran, T., \& Sari, R. 2013, ApJ, 764, 179

Bruzual, G., \& Charlot, S. 2003, MNRAS, 344, 1000

Burrows, D. N., Grupe, D., Capalbi, M., et al. 2006, ApJ, 653, 468

Burrows, D. N., Hill, J. E., Nousek, J. A., et al. 2005, SSRv, 120, 165

Cannizzo, J. K., Barbier, L. M., Barthelmy, S. D., et al. 2006, GCNR, 20, 1

Cardelli, J. A., Clayton, G. C., \& Mathis, J. S. 1989, ApJ, 345, 245

Castro-Tirado, A. J., de Ugarte Postigo, A., Gorosabel, J., et al. 2005, A\&A, 439, L15

Cenko, S. B., Berger, E., Nakar, E., et al. 2008, arXiv:0802.0874

Chapman, R., Levan, A. J., Wynn, G. A., et al. 2008, in AIP Conf. Ser. 983, 40 Years of Pulsars: Millisecond Pulsars, Magnetars and More, ed. C. Bassa, Z. Wang, A. Cumming, \& V. M. Kaspi (Melville, NY: AIP), 301 Christensen, L., Hjorth, J., \& Gorosabel, J. 2004, A\&A, 425, 913 Cummings, J., Barbier, L., Barthelmy, S., et al. 2005, GCN, 4365, 1 D'Avanzo, P., Fiore, F., Piranomonte, S., et al. 2007, GCN, 7152, 1 D’ Avanzo, P., Malesani, D., Covino, S., et al. 2009, A\&A, 498, 711 de Pasquale, M., Markwardt, C., \& Sbarufatti, B. 2010, GCNR, 269, 1 de Ugarte Postigo, A., Castro-Tirado, A. J., Guziy, S., et al. 2006, ApJL, 648, L83

Djorgovski, S. G., Kulkarni, S. R., Bloom, J. S., et al. 1998, ApJL, 508, L17

Dominik, M., Belczynski, K., Fryer, C., et al. 2012, ApJ, 759, 52

Driver, S. P., Allen, P. D., Liske, J., \& Graham, A. W. 2007, ApJL, 657, L85

Eichler, D., Livio, M., Piran, T., \& Schramm, D. N. 1989, Natur, 340, 126

Evans, P. A., Beardmore, A. P., Page, K. L., et al. 2009, MNRAS, 397, 1177

Evans, P. A., Goad, M. R., Osborne, J. P., \& Beardmore, A. P. 2011, GCN, 11556,1

Foley, R. J., Bloom, J. S., \& Chen, H.-W. 2005, GCN, 3808, 1

Fong, W., Berger, E., Chornock, R., et al. 2011, ApJ, 730, 26

Fong, W., Berger, E., \& Fox, D. B. 2010, ApJ, 708, 9

Fong, W., Berger, E., Margutti, R., et al. 2012, ApJ, 756, 189

Fox, D. B., Frail, D. A., Price, P. A., et al. 2005, Natur, 437, 845

Fruchter, A. S., Levan, A. J., Strolger, L., et al. 2006, Natur, 441, 463

Fryer, C. L., Woosley, S. E., \& Hartmann, D. H. 1999, ApJ, 526, 152

Gal-Yam, A., Nakar, E., Ofek, E. O., et al. 2008, ApJ, 686, 408

Gehrels, N., Chincarini, G., Giommi, P., et al. 2004, ApJ, 611, 1005

Gehrels, N., Sarazin, C. L., O'Brien, P. T., et al. 2005, Natur, 437, 851

Gelbord, J. M., Barthelmy, S. D., Chester, M. M., et al. 2010, GCN, 11461, 1

Gelbord, J. M., \& Grupe, D. 2010, GCN, 11474, 1

Goad, M. R., Tyler, L. G., Beardmore, A. P., et al. 2007, A\&A, 476, 1401 Golenetskii, S., Aptekar, R., Frederiks, D., et al. 2010a, GCN, 10890, 1 Golenetskii, S., Aptekar, R., Frederiks, D., et al. 2010b, GCN, 11470, 1 Gotz, D., Beckmann, V., Mereghetti, S., \& Paizis, A. 2007, GCN, 6607, 1 Granot, J., \& Sari, R. 2002, ApJ, 568, 820

Grupe, D., Burrows, D. N., Patel, S. K., et al. 2006, ApJ, 653, 462 
Hakobyan, A. A., Petrosian, A. R., McLean, B., et al. 2008, A\&A, 488, 523 Hjorth, J., Sollerman, J., Gorosabel, J., et al. 2005a, ApJL, 630, L117 Hjorth, J., Watson, D., Fynbo, J. P. U., et al. 2005b, Natur, 437, 859

Holland, S. T., Burrows, D. N., Kennea, J. A., et al. 2010a, GCN, 10884, 1

Holland, S. T., Landsman, W. B., Page, K. L., \& Stamatikos, M. 2010b, GCNR, 289,1

Hoversten, E. A., Krimm, H. A., Grupe, D., et al. 2009, GCNR, 218, 1

Ilbert, O., Salvato, M., Le Floc'h, E., et al. 2010, ApJ, 709, 644

Immler, S., Starling, R. L. C., Evans, P. A., Barthelmy, S. D., \& Sakamoto, T. 2010, GCNR, 290, 1

Kalberla, P. M. W., Burton, W. B., Hartmann, D., et al. 2005, A\&A, 440,775

Kelly, P. L., \& Kirshner, R. P. 2012, ApJ, 759, 107

Kennicutt, R. C., Jr. 1998, ARA\&A, 36, 189

Kochanek, C. S., Pahre, M. A., Falco, E. E., et al. 2001, ApJ, 560, 566

Kodaka, N., Tashiro, M., Urata, Y., et al. 2007, GCN, 6637, 1

Kouveliotou, C., Meegan, C. A., Fishman, G. J., et al. 1993, ApJL, 413, L101

Krimm, H., Barbier, L., Barthelmy, S., et al. 2005, GCN, 3667, 1

Krimm, H. A., Barthelmy, S. D., Baumgartner, W. H., et al. 2008, GCN, 8735,1

Krimm, H. A., Barthelmy, S. D., Baumgartner, W. H, et al. 2010a, GCN, 11467,1

Krimm, H. A., Barthelmy, S. D., Beardmore, A. P., et al. 2009, GCN, 8936, 1

Krimm, H. A., Cummings, J. R., Evans, P. A., \& Marshall, F. E. 2010b, GCNR, 271,1

Kuin, N. P. M., \& Gelbord, J. M. 2010, GCN, 11472, 1

La Parola, V., Mangano, V., Fox, D., et al. 2006, A\&A, 454, 753

Laskar, T., Berger, E., \& Chary, R.-R. 2011, ApJ, 739, 1

Le Floc'h, E., Duc, P.-A., Mirabel, I. F., et al. 2003, A\&A, 400, 499

Leibler, C. N., \& Berger, E. 2010, ApJ, 725, 1202

Levan, A. J., Tanvir, N. R., Fruchter, A. S., et al. 2006a, ApJL, 648, L9

Levan, A. J., Tanvir, N. R., Hjorth, J., et al. 2009, GCN, 10154, 1

Levan, A. J., Wynn, G. A., Chapman, R., et al. 2006b, MNRAS, 368, L1

Levesque, E. M., Bloom, J. S., Butler, N. R., et al. 2010, MNRAS, 401, 963

Li, W., Chornock, R., Leaman, J., et al. 2011, MNRAS, 412, 1473

Mannucci, F., Della Valle, M., Panagia, N., et al. 2005, A\&A, 433, 807

Mao, J., Guidorzi, C., Ukwatta, T., et al. 2008, GCNR, 138, 1

Maoz, D., Mannucci, F., \& Brandt, T. D. 2012, MNRAS, 426, 3282

Maoz, D., Sharon, K., \& Gal-Yam, A. 2010, ApJ, 722, 1879

Maraston, C. 2005, MNRAS, 362, 799

Margutti, R., Berger, E., Fong, W., et al. 2012, ApJ, 756, 63

Margutti, R., Genet, F., Granot, J., et al. 2010, MNRAS, 402, 46

Margutti, R., Zaninoni, E., Bernardini, M. G., et al. 2013, MNRAS, 428, 729

Markwardt, C., Barbier, L., Barthelmy, S., et al. 2006, GCN, 4873, 1

Markwardt, C., Beardmore, A., Marshall, F. E., et al. 2007, GCNR, 51, 1

Marshall, F., Perri, M., Stratta, G., et al. 2006, GCNR, 18, 1

Marshall, F. E., Barthelmy, S. D., Burrows, D. N., et al. 2007, GCNR, 80, 1

McBreen, S., Krühler, T., Rau, A., et al. 2010, A\&A, 516, A71

Metzger, B. D., Quataert, E., \& Thompson, T. A. 2008, MNRAS, 385, 1455

Naito, H., Sako, T., Suzuki, D., et al. 2010, GCN, 10889, 1

Nakar, E., Gal-Yam, A., \& Fox, D. B. 2006, ApJ, 650, 281

Narayan, R., Paczynski, B., \& Piran, T. 1992, ApJL, 395, L83
Nicuesa Guelbenzu, A., Klose, S., Greiner, J., et al. 2012, A\&A, 548, A101 Oates, S. R., Page, K. L., Evans, P. A., \& Markwardt, C. B. 2009, GCNR, 259,1

Oemler, A., Jr., \& Tinsley, B. M. 1979, AJ, 84, 985

O'Shaughnessy, R., Belczynski, K., \& Kalogera, V. 2008, ApJ, 675, 566

Pagani, C., Racusin, J. L., Brown, P., \& Cummings, J. 2008, GCNR, 162, 1

Page, K. L., \& Holland, S. T. 2010, GCN, 10888, 1

Panaitescu, A., Kumar, P., \& Narayan, R. 2001, ApJL, 561, L171

Perley, D. A., Metzger, B. D., Granot, J., et al. 2009, ApJ, 696, 1871

Perley, D. A., Modjaz, M., Morgan, A. N., et al. 2012, ApJ, 758, 122

Piranomonte, S., D’Avanzo, P., Covino, S., et al. 2008, A\&A, 491, 183

Poole, T. S., Breeveld, A. A., Page, M. J., et al. 2008, MNRAS, 383, 627

Predehl, P., \& Schmitt, J. H. M. M. 1995, A\&A, 293, 889

Prochaska, J. X., Bloom, J. S., Chen, H.-W., et al. 2006, ApJ, 642, 989

Qin, B., Wu, X.-P., Chu, M.-C., Fang, L.-Z., \& Hu, J.-Y. 1998, ApJL, 494, L57

Racusin, J., Barbier, L., \& Landsman, W. 2007, GCNR, 70, 1

Roming, P. W. A., Vanden Berk, D., Pal'shin, V., et al. 2006, ApJ, 651, 985

Rowlinson, A., Wiersema, K., Levan, A. J., et al. 2010, MNRAS, 408, 383

Sakamoto, T., Barthelmy, S. D., Baumgartner, W. H., et al. 2011, GCN, 12464,1

Sakamoto, T., Norris, J., Ukwatta, T., et al. 2007, GCN, 7156, 1

Sakamoto, T., Troja, E., Aoki, K., et al. 2013, ApJ, 766, 41

Sari, R., Piran, T., \& Halpern, J. P. 1999, ApJL, 519, L17

Sato, G., Angelini, L., Barbier, L., et al. 2005, GCN, 3793, 1

Sato, G., Barbier, L., Barthelmy, S., et al. 2006a, GCN, 5064, 1

Sato, G., Barbier, L., Barthelmy, S. D., et al. 2006b, GCN, 5381, 1

Sato, G., Barbier, L., Barthelmy, S. D., et al. 2007a, GCN, 6681, 1

Sato, G., Barbier, L., Barthelmy, S. D., et al. 2007b, GCN, 7148, 1

Savaglio, S., Glazebrook, K., \& Le Borgne, D. 2009, ApJ, 691, 182

Schady, P., Breeveld, A., Poole, T. S., et al. 2006, GCNR, 6, 1

Schlafly, E. F., \& Finkbeiner, D. P. 2011, ApJ, 737, 103

Schlegel, D. J., Finkbeiner, D. P., \& Davis, M. 1998, ApJ, 500, 525

Siegel, M. H., Grupe, D., de Pasquale, M., et al. 2010, GCNR, 292, 1

Soderberg, A. M., Berger, E., Kasliwal, M., et al. 2006, ApJ, 650, 261

Stamatikos, M., Beardmore, A. P., Cummings, J. R., et al. 2011, GCN, 11553, 1

Stanford, S. A., Dickinson, M., Postman, M., et al. 2004, AJ, 127, 131

Stratta, G., D'Avanzo, P., Piranomonte, S., et al. 2007, A\&A, 474, 827

Sullivan, M., Le Borgne, D., Pritchet, C. J., et al. 2006, ApJ, 648, 868

Suzuki, D., Hayashi, F., Kobara, S., et al. 2010, GCN, 10885, 1

Tamura, N., \& Ohta, K. 2004, MNRAS, 355, 617

Uehara, T., Ohno, M., Takahashi, T., et al. 2008, GCN, 7223, 1

Ukwatta, T. N., Tueller, J., Mangano, V., et al. 2008, GCNR, 111, 1

Urata, Y., Tashiro, M., Abe, K., et al. 2006, GCN, 5717, 1

van den Bergh, S., Li, W., \& Filippenko, A. V. 2005, PASP, 117, 773

Villasenor, J. S., Lamb, D. Q., Ricker, G. R., et al. 2005, Natur, 437, 855

Wainwright, C., Berger, E., \& Penprase, B. E. 2007, ApJ, 657, 367

Wakker, B. P., Lockman, F. J., \& Brown, J. M. 2011, ApJ, 728, 159

Watson, D. 2011, A\&A, 533, A16

Xin, L. P., Zhang, T. M., Qiu, Y. L., et al. 2011, GCN, 11554, 1

Zheng, Z., \& Ramirez-Ruiz, E. 2007, ApJ, 665, 1220

Ziaeepour, H., Barthelmy, S., Burrows, D. N., et al. 2006, GCNR, 21, 2

Ziaeepour, H., Barthelmy, S. D., Parsons, A., et al. 2007, GCNR, 74, 2 